\title{
4-1BB enhancement of CAR T function requires NF-KB and TRAFs
}

\author{
Gongbo Li, Justin C. Boucher, ${ }^{1}$ Hiroshi Kotani, ${ }^{1}$ Kyungho Park, ${ }^{1}$ Yongliang Zhang, ${ }^{1}$ \\ Bishwas Shrestha, ${ }^{1}$ Xuefeng Wang, ${ }^{2}$ Lawrence Guan, ${ }^{3}$ Nolan Beatty, ${ }^{3}$ Daniel Abate-Daga, ${ }^{3,4,5}$ \\ and Marco L. Davila $a^{3,5,6}$ \\ ${ }^{1}$ Clinical Science Division, H. Lee Moffitt Cancer Center and Research Institute, Tampa, Florida, USA. ${ }^{2}$ Department of \\ Biostatistics and Bioinformatics, H. Lee Moffitt Cancer Center and Research Institute, Tampa, Florida, USA. ${ }^{3}$ Morsani \\ College of Medicine, University of South Florida, Tampa, Florida, USA. ${ }^{4}$ Department of Cutaneous Oncology, H. Lee Moffitt \\ Cancer Center and Research Institute, Tampa, Florida, USA. ${ }^{5}$ Department of Immunology, H. Lee Moffitt Cancer Center and \\ Research Institute, Tampa, Florida, USA. ${ }^{6}$ Department of Blood and Marrow Transplantation and Cellular Immunotherapy, \\ H. Lee Moffitt Cancer Center and Research Institute, Tampa, Florida, USA.
}

Chimeric antigen receptors (CARs) have an antigen-binding domain fused to transmembrane, costimulatory, and $\mathrm{CD} 3 \zeta$ domains. Two CARs with regulatory approval include a $\mathrm{CD28}$ or 4-1BB costimulatory domain. While both CARs achieve similar clinical outcomes, biologic differences have become apparent but not completely understood. Therefore, in this study we aimed to identify mechanistic differences between 4-1BB and CD28 costimulation that contribute to the biologic differences between the 2 CARs and could be exploited to enhance CAR T cell function. Using CD19-targeted CAR T cells with 4-1BB we determined that enhancement of $T$ cell function is driven by NF- $\kappa B$. Comparison to CAR T cells with CD28 also revealed that 4-1BB is associated with more antiapoptotic proteins and dependence on persistence for $B$ cell killing. While TNF receptorassociated factor 2 (TRAF2) has been presupposed to be required for 4-1BB costimulation in CAR T cells, we determined that TRAF1 and TRAF3 are also critical. We observed that TRAFs impacted CAR T viability and proliferation, as well as cytotoxicity and/or cytokines, in part by regulating NFкB. Our study demonstrates how 4-1BB costimulation in CAR T cells impacts antitumor eradication and clinical outcomes and has implications for enhanced CAR design.

Conflict of interest: MLD is the inventor of patents (application no. 62/666,381 and PCT/US2018/042470) relevant to the work described in this manuscript. In addition, H. Lee Moffitt Cancer Center and Research Institute has filed patents on the technology described in the manuscript, which has been licensed to Atara Biotherapeutics.

Submitted: March 27, 2018

Accepted: August 7, 2018

Published: September 20, 2018

\section{Reference information:}

JCI Insight. 2018;3(18):e121322.

https://doi.org/10.1172/jci.

insight.121322.

\section{Introduction}

Adoptive $\mathrm{T}$ cell transfer as a therapy has generated excitement due to the induction of durable responses in patients with chemotherapy-refractory cancer (1-4). The development of autologous tumor-specific $\mathrm{T}$ cells as a cancer therapy began nearly 30 years ago (1) with isolation and expansion of tumor-infiltrating lymphocytes (TILs), but the first clinical approval occurred in 2017 and was for autologous T cells genetically modified to express a chimeric antigen receptor (CAR). The CAR is a hybrid antigen receptor composed of the intracellular signaling and activation domains of the $\mathrm{T}$ cell receptor (TCR) and extracellular antigen-binding domain, or single-chain variable fragment (scFv), derived from an antibody (5). The CAR is expressed on the surface of $\mathrm{T}$ cells by inclusion of hinge and transmembrane domains. The CAR represented a major scientific advance from TILs for several reasons, including that CARs are modular receptors, so different components can be added to provide new $\mathrm{T}$ cell functions. The most common modifications have been the addition of costimulatory domains, such as CD28 or $4-1 \mathrm{BB}$, that represent second-generation CAR designs and are the largest CAR class under clinical evaluation. First-generation CARs, which contain the $\mathrm{CD} 3 \zeta$ activation domain paired to a transmembrane domain and $\mathrm{scFv}$, were sufficient to re-target primary $\mathrm{T}$ cells in vitro, but insufficient in vivo (6, 7). However, CD28 costimulation of first-generation CAR T cells by CD80 ligation supported robust cytokine production and proliferation in vitro, and enhanced CAR T cell persistence and tumor killing in vivo $(7,8)$. Furthermore, preclinical studies of $\mathrm{T}$ cells gene-targeted with second-generation CARs, including costimulatory domains, such as CD28 or 4-1BB, and CD3 This validation of the antitumor efficacy of second-generation CAR T cells served as the preclinical rationale for their clinical evaluation. 
Clinical trials with second-generation CAR T cells have identified efficacious response rates for B cell malignancies, such as B cell acute lymphoblastic leukemia (B-ALL), diffuse large B cell lymphoma (DLBCL), and other non-Hodgkin lymphoma (NHL) (14-23). However, as the number of patients treated with CD19-targeted CAR T cells and follow-up has increased, low response rates for NHL and high relapse rates for B-ALL are major obstacles to long-term efficacy and are an important focus for refinement. An option for improvement in CAR T cell function is optimization of costimulation. While the initial response rates are similar between second-generation CAR T cells that contain a CD28 (h1928z) or 4-1BB (h19BBz) costimulatory domain, they contribute to clinical outcomes differently. For example, lack of persistence correlates with relapse after treatment of B-ALL with h19BBz CAR T cells (15). In contrast, response associated with h1928z CAR T cells for B-ALL does not strictly depend on persistence since in some trials the CAR T cells do not persist more than 40 days (14-16).

As research is being directed towards optimization of second-generation CAR T cells to enhance efficacy or decrease relapse, we aimed to identify how 4-1BB costimulatory signaling enhances CAR T cell function. In this study, we identify NF- $\mathrm{KB}$ as a critical signaling pathway for 4-1BB costimulation in second-generation $\mathrm{CAR} \mathrm{T}$ cells and it contributes to increased proliferation and/or persistence, which supports efficacious eradication of leukemia. Furthermore, by using dominant-negative (DN) inhibitors of TNF receptor-associated factor (TRAF) proteins we demonstrate that TRAF1 and TRAF3 are required for optimal NF- $\mathrm{kB}$ signaling by CARs with a 4-1BB costimulatory domain, which is consistent with prior research involving non-engineered $\mathrm{T}$ cells $(24,25)$. Considering that detailed studies have not been performed in $\mathrm{T}$ cells expressing CARs with 4-1BB endodomains, we also translated our observations for the role of TRAF proteins and NF- $\kappa B$ from primary mouse CAR T cells to human CAR T cells. By overexpressing TRAFs we enhanced 4-1BB-based human CAR T cell viability, proliferation, and cytotoxicity. Thus, our studies provide a rationale for refinement of CAR design by enhancing TRAF protein recruitment and NF- $\mathrm{KB}$ signaling.

\section{Results}

At stress dose levels CD19-targeted CAR T cells with a mouse 4-1BB endodomain eradicate leukemia less efficaciously than $T$ cells with a CAR containing a CD28 endodomain. We evaluated 4 mouse CD19-targeted (mCD19targeted) CARs, which are all murine derived with the same extracellular rat-origin anti-mCD19 scFv paired to mouse CD $8 \alpha$ hinge and transmembrane domains. They differ only in their intracellular activation and costimulatory domains by including no domains $(\mathrm{m} 19 \Delta \mathrm{z}), \mathrm{CD} 3 \zeta$ alone $(\mathrm{m} 19 \mathrm{z})$, or $\mathrm{CD} 3 \zeta$ paired with the CD28 (m1928z) or the 4-1BB costimulatory domain (m19-musBBz). We performed a comparison of mCD19-targeted CAR T cells in an immune-competent mouse model (26) with the rationale that this will allow us to identify biologic differences in adoptively transferred CAR T cells mediated by costimulation, which could be further investigated to identify signaling mechanisms driving these differences. We evaluated antigen-specific cytotoxicity of these mouse CAR T cells in a 4-hour chromium-release assay, which demonstrated that $\mathrm{m} 1928 \mathrm{z}$ or $\mathrm{m} 19 \mathrm{z}$ CAR T cells lysed CD19+ target cells at similar levels, while m19-mus$\mathrm{BBz}$ CAR T cells were less efficacious (Figure 1A). After overnight stimulation with 3T3-mCD19 artificial antigen-presenting cells (AAPCs), m1928z CAR T cells released greater amounts of IFN- $\gamma$ and TNF- $\alpha$ than m19-musBBz CAR T cells (Figure 1B).

We next compared the in vivo function of mCD19-targeted CAR T cells using our B-ALL mouse model (26). C57BL/6 mice were intravenously (i.v.) injected with E $\mu$-ALL cells and 1 week later mice were treated with intraperitoneal (i.p.) cyclophosphamide followed by mCD19-targeted CAR T cells. Despite less efficacious in vitro function, at a dose of $5 \times 10^{6}$ cells (Figure 1C and Supplemental Figure 1A; supplemental material available online with this article; https://doi.org/10.1172/jci.insight.121322DS1) m19-musBBz CAR T cells supported survival similar to that of m1928z CAR T cells. Both m1928z and m19-musBBz CAR T cells maintained B cell aplasia and had comparable persistence in the peripheral blood 3 weeks after infusion (Figure 1D). To increase our ability to detect small differences in efficacy between CARs we performed a stress test as previously described (27) and titrated T cell doses down to levels that had difficulty sustaining B cell aplasia and CAR T cell persistence (Supplemental Figure 1B). At the $3 \times 10^{5}$ dose, only 1 out of 4 mice treated with m1928z CAR T cells maintained B cell aplasia 3 weeks after injection (Supplemental Figure 1B). Therefore, we chose this, or lower doses, to compare in vivo CAR T cell function. At this lower stress-test dose, m1928z CAR T cells provided superior protection against leukemia compared with m19-musBBz or m19z CAR T cells (Figure 1E). Also, m1928z CAR T cells had enhanced in vivo B cell aplasia and donor $\mathrm{T}$ cell persistence compared with m19-musBBz (Figure 1F). 
A
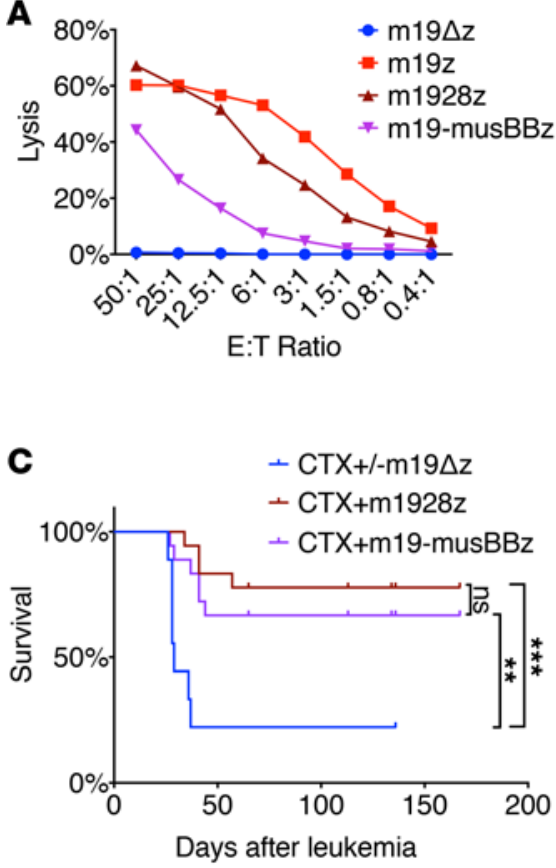

E

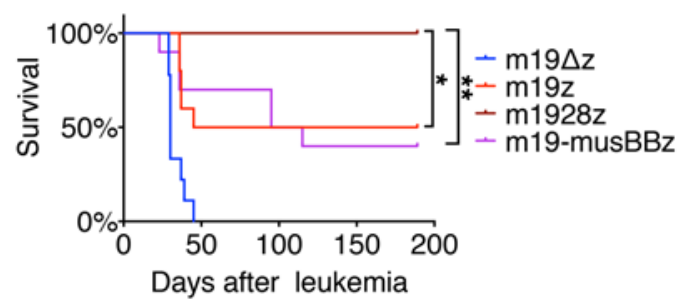

B

D
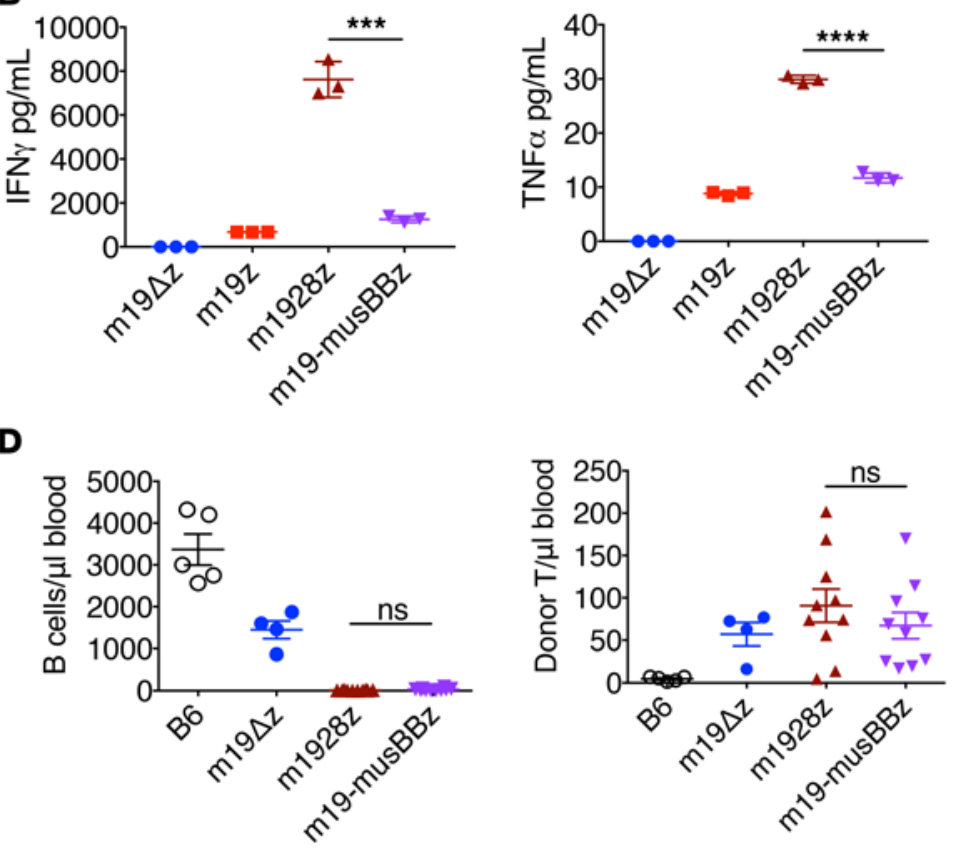

$\mathbf{F}$

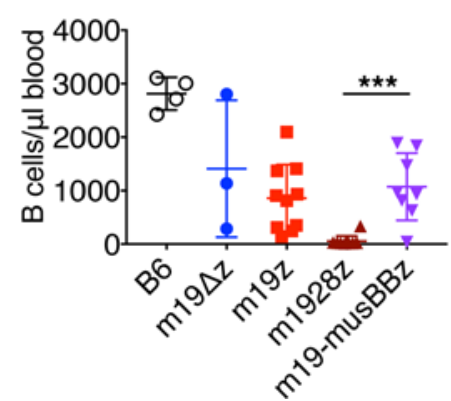

Figure 1. Comparison of mouse T cells with mCD19-targeted CARs having different intracellular domains. (A) Cytotoxicity assay. CAR T cells were cocultured with EL4-mCD19 cells at indicated E/T ratios. Cytotoxicity was evaluated with a chromium-release assay. Data are representative of 3 independent experiments in triplicate. (B) Cytokine production. CAR T cells were cocultured with 3T3-mCD19 cells for 24 hours. Supernatants were collected for Luminex assay. Data are representative of 2 independent experiments in triplicate. (C) Survival and (D) in vivo B cell killing and T cell persistence 3 weeks after CAR T injection at $5 \times 10^{6}$ dose. Six days after injection with $\mathrm{E} \mu$-ALL cells, mice were i.p. injected with cyclophosphamide (CTX) followed 1 day later with an i.v. injection of $5 \times 10^{6} \mathrm{~T}$ cells. Survival data are pooled from 2 independent experiments $(n=45$ total). Negative control groups are CTX alone or with m $19 \Delta z$ CAR T cells (CTX $\pm m 19 \Delta z)$. (E) Survival and (F) in vivo B cell killing and T cell persistence 4 weeks after CAR T injection at $3 \times 10^{5} T$ cell dose. Seven days after injection with $\mathrm{E} \mu-\mathrm{ALL}$, mice were i.p. injected with CTX followed 1 day later with an i.v. injection of CAR T cells. Survival data are from 1 experiment ( $n=39$ total). B (B220+CD19 $)$ and donor T (CD3+Thy1.1 ${ }^{+}$) cells in the blood were quantified using CountBright counting beads. For $\mathbf{D}$ and $\mathbf{F}$, each data point represents 1 mouse. ${ }^{*} P<0.05 ;{ }^{* *} P<0.01 ;{ }^{* *} P<0.001$; ${ }^{* * *} P<0.0001$ by log-rank test (C and E) or unpaired $t$ test (B, D, and F). ns, not significant.

We evaluated the gene expression by microarray of sorted mCD19-targeted CAR T cells after stimulation with 3T3-mCD19 AAPCs to determine how gene expression, and signaling pathways, were impacted by costimulation in mouse CAR T cells. Since CAR T cells can downregulate the CAR after ligation (28), we modified the CARs to be directly conjugated to a fluorescent protein using a glycineserine linker after $\mathrm{CD} 3 \zeta$ in lieu of a reporter not directly associated with the CAR to exclude sorting and analysis of CAR-negative T cells (Supplemental Figure 2A). mCD19-targeted CAR T cells with a fluorescent protein tag showed reproducible patterns of CAR expression (Supplemental Figure 2B). A total of 205 genes were found to be differentially expressed by m19-musBBz CAR T cells compared with $\mathrm{m} 19 \mathrm{z}$ and $\mathrm{m} 1928 \mathrm{z}$ CAR T cells (Supplemental Figure 2, C-E). These include the upregulation of effector genes (Gzmf, Ifng, Prf1), as well as exhaustion genes or transcription factors (Havcr2, CD244, Klrg1, Eomes) in $\mathrm{m} 19 \mathrm{z}$ and $\mathrm{m} 1928 \mathrm{z}$ CAR T cells (Supplemental Tables 1-4). In contrast, m19-musBBz CAR $\mathrm{T}$ cells upregulate genes critical for NF-kB regulation, $\mathrm{T}$ cell quiescence, and memory (Fos, Jun, Tcf7, 
NF-кBia, K1f2/4). We also observed that cytokine production, immune phenotype, as well as in vivo leukemia eradication, B cell killing, and persistence were not significantly impacted by the fluorescent reporter (Supplemental Figure 3, A-C). We followed the temporal kinetics of CAR T and B cell numbers in the blood to evaluate CAR T cell persistence using the fluorescently tagged CARs. By 1 week of adoptive transfer there were already differences between CAR T and B cell numbers in the blood (Supplemental Figure 3D). This persisted up to week 4 after adoptive transfer, with B cell numbers still being different but CAR T cell numbers starting to become similar. Therefore, we chose to focus our analyses of CAR T cell persistence in the blood at the time points between 1 and 4 weeks after adoptive transfer.

Inclusion of the human 4-1BB endodomain in mCD19-targeted CAR T cells enhances in vivo function. Clinical results $(14-17,20,22)$ have demonstrated similar efficacious complete response rates for patients with B-ALL when treated with second-generation human CAR T cells that include a CD28 or 4-1BB endodomain. However, using stress-test dosing in our mouse model the $\mathrm{m} 19-\mathrm{musBBz}$ appeared modestly less efficacious than m1928z, even though there was evidence of 4-1BB costimulation (Supplemental Figure 2, C-E). We speculated that sequence differences between human and mouse $4-1 \mathrm{BB}$ endodomains, which are $54 \%$ identical (Figure 2A), contribute to the modestly reduced efficacy that is not consistent with clinical observations of second-generation CAR T cells. Previous studies have demonstrated that both mouse and human 4-1BB endodomains bind TRAFs, which enhance signaling downstream of TNF-receptor-family proteins such as 4-1BB (29-33). However, in vitro assays $(29,30)$ suggest that human 4-1BB binds TRAF1-3 while mouse 4-1BB binds only TRAF1 and -2, leading us to hypothesize that substituting human 4-1BB in mCD19-targeted CAR T cells may enhance TRAF3 binding and CAR T cell function (34). Furthermore, comparison of 4-1BB endodomains with differential TRAF binding abilities may allow us to identify signaling pathways that support enhanced in vivo function by CAR T cells that rely on 4-1BB costimulation. Therefore, we created a variant CAR (m19-humBBz) that included the human 4-1BB endodomain paired with the murine scFv, CD8, and CD3 $\zeta$ domains included in the other anti-mouse CD19 CARs (Supplemental Figure 2A).

We compared the in vitro function of m19-humBBz CAR T cells with other CD19-targeted CAR T cells. After 4-hour stimulation with 3T3-mCD19 AAPCs, intracellular flow cytometry demonstrated that $\mathrm{m} 1928 \mathrm{z} \mathrm{CD} 8^{+} \mathrm{CAR}$ T cells were $8.4 \%$ positive for IFN- $\gamma$, which is significantly greater than m19-humBBz (1.4\%) or m19-musBBz CD8 ${ }^{+}$CAR T cells (average $0.3 \%$ ) (Figure $2 \mathrm{~B}$ ). In addition, m1928z CAR T cells produced the greatest amount of TNF- $\alpha$. There was also enhancement (approximately 2-fold) of TNF- $\alpha$ production by $\mathrm{T}$ cells modified with the m19-humBBz CAR compared with the m19-musBBz CAR (Figure 2B). A cytotoxicity assay at an effector/target (E/T) ratio of 10:1 demonstrated that $\mathrm{T}$ cells modified with the m19-humBBz CAR did not have enhanced killing compared with m19-musBBz and were less efficacious than $\mathrm{m} 19 \mathrm{z}$ and $\mathrm{m} 1928 \mathrm{z}$ CARs, which killed all target cells rapidly (Figure 2C).

We also compared the in vivo function of m19-humBBz CAR T cells with other mCD19-targeted CAR T cells in our E $\mu$-ALL model using a stress-test cell dose. CAR T cells among all the groups had similarly balanced CD4/CD8 ratios and an immune phenotype composed mostly of central memory $\left(\mathrm{CD} 44^{+} \mathrm{CD} 62 \mathrm{~L}^{+}\right) \mathrm{T}$ cells (Supplemental Figure 4). As expected, overall survival (OS) was poor in $\mathrm{m} 19 \Delta \mathrm{z}$ and $\mathrm{m} 19 \mathrm{z}$ CAR $\mathrm{T}$ cell groups compared with OS imparted by m1928z CAR T cells, which was $57 \%$ at day 150 (Figure 2D). However, despite their poor in vitro function, under stress-test conditions m19-humBBz CAR T cells mediated an OS of $70 \%$ at day 150 , which was significantly enhanced compared with $\mathrm{m} 19 \Delta \mathrm{z}$ and $\mathrm{m} 19 \mathrm{z}$ CAR $\mathrm{T}$ cells and similar to m1928z CAR T cells (Figure 2D). The improved survival mediated by m19-humBBz and m1928z CAR T cells was reflected by B and CAR $\mathrm{T}$ cells in the femurs of treated mice. One week after treatment, both m19-humBBz and m1928z CAR $\mathrm{T}$ cells had similar persistence in the bone marrow (BM) and induced B cell aplasia to a significantly greater extent than $\mathrm{m} 19 \mathrm{z}$ CAR T cells (Figure 2E).

m19-humBBz CAR T cells rely on persistence to enhance function in vivo. It was recently reported (27) that 4-1BB costimulation in human CAR $\mathrm{T}$ cells supported enhanced persistence in immune-deficient mice. Therefore, we hypothesized that equivalent in vivo anti-leukemia killing by $\mathrm{m} 19$-humBBz and $\mathrm{m} 1928 \mathrm{z}$ CAR T cells, despite differential in vitro function, was due to enhanced m19-humBBz CAR T cell persistence secondary to $4-1 \mathrm{BB}$ costimulation. Our rationale for evaluating this hypothesis was that identification of a signaling pathway contributing to enhanced persistence would allow potentiation of this attribute. However, we did not identify differences in second-generation CAR T cell persistence in our model (Figure 2E). Therefore, we evaluated in vivo expansion of mCD19-targeted CAR T cells in Rag1 ${ }^{-1-}$ mice, which lack the $\mathrm{mCD} 19$ antigen, since the prior report (27) demonstrating enhanced persistence was performed 
A hum41BB IC domain 1 KRGRKKLLYIFKQPFMRPVQTTQEEDGCSCRFPEEEE---GGCEL 42 mus41BB IC domain 1 KWIRKKEPHIFKQPFKKTTGAAQEEDACSCRCPQEEEGGGGGYEL 45

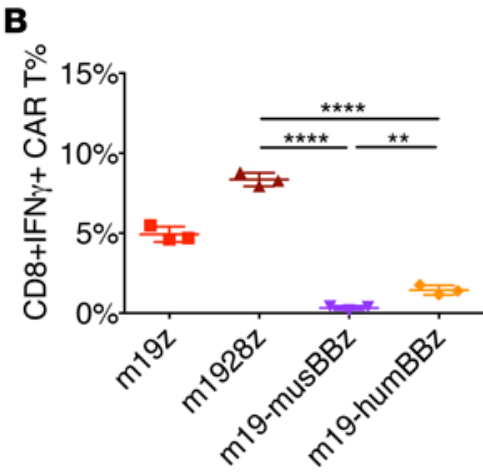

$* * * \quad * * * * * *$

$* * * * * * * * * * * * \quad * * * *$

\section{5}
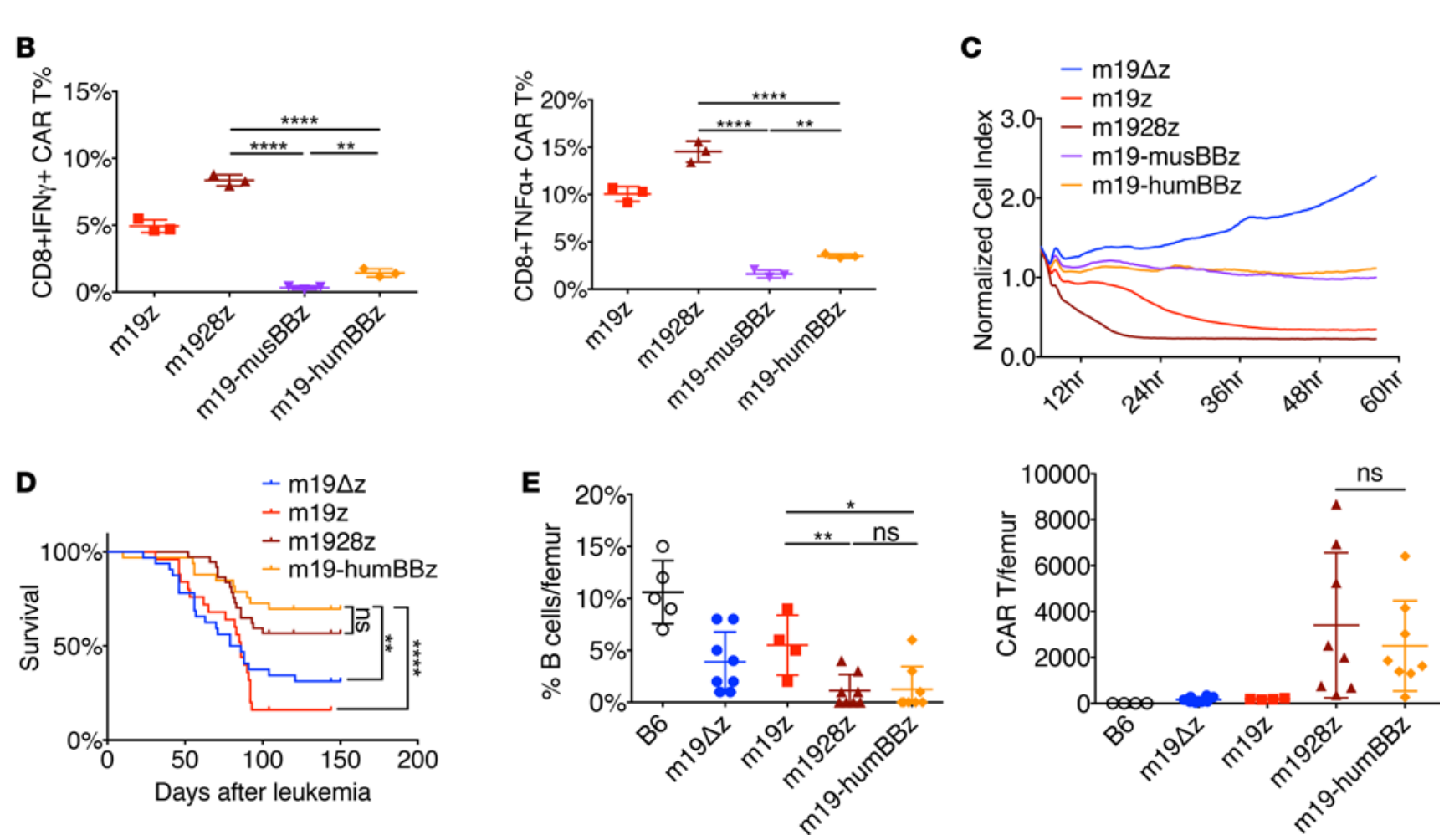

Figure 2. At a stress-test dose, mCD19-targeted CAR T cells containing a human 4-1BB endodomain (m19-humBBz) display comparable in vivo function to $\mathbf{m} 1928 z$ CAR T cells. (A) Amino acid sequence alignment of mouse and human 4-1BB endodomains. Identical amino acids are indicated with an asterisk. (B) Intracellular IFN- $\gamma$ and TNF- $\alpha$ in CAR T cells upon mCD19 antigen stimulation. One million transduced T cells were cocultured with $1 \times 10^{5}$ irradiated 3T3-mCD19 for 4 hours in the presence of protein transport inhibitor. Cells were analyzed by flow cytometry. Data are representative of 2 independent experiments performed in triplicate. (C) Cytotoxicity assay. CAR T cells were cocultured with 3T3-mCD19 at an E/T ratio of 10:1 and target cell killing was monitored on an xCELLigence RTCA (real-time cell analysis) system. Data are from 1 experiment in triplicate. (D) Overall survival. Mice were treated i.p. with cyclophosphamide (250-300 mg $/ \mathrm{kg})$ and i.v. CAR T cells $\left(1.5 \times 10^{5}\right.$ to $3 \times 10^{5}$ CAR T cells per mouse). Data are pooled from 4 independent experiments $(n=127)$. (E) B (CD19+B220+) and CAR T (CD3+CAR+) cells in femurs 1 week after CAR T cell injection. Bone marrow cells were isolated and analyzed by flow cytometry. Data are pooled from 2 independent experiments $(n=33$ total). Each data point indicates 1 mouse. All counts were calculated with CountBright beads. ${ }^{*} P<0.05 ;{ }^{* *} P<0.01 ;{ }^{* * *} P<0.0001$ by unpaired $t$ test (B and $\mathbf{E}$ ) or log-rank test (D). ns, not significant.

in immune-deficient mice. $R a g 1^{-/-}$mice were i.v. injected with $1 \times 10^{6} \mathrm{CAR}$ T cells and $\mathrm{BM}$ was isolated 1 week later. The m19-humBBz CAR T cells had the greatest in vivo persistence at 1.5 -fold greater than m1928z CAR T cells (Figure 3A).

To determine if $\mathrm{m} 19$-humBBz CAR $\mathrm{T}$ cells required persistence for optimal function in vivo we irradiated CAR T cells (10 Gy) prior to injection. C57BL/6 mice were i.p. injected with cyclophosphamide followed by CAR T cells (Supplemental Figure 5) 1 day later. CAR T cell persistence and B cell killing in peripheral blood and BM were evaluated 1 week after CAR T cell transfer. In both the blood and BM, irradiation significantly reduced persistence of m19-humBBz but not m1928z CAR T cells (Figure 3B). Correspondingly, in blood there was early B cell recovery in all mice in the irradiated m19-humBBz group compared with 9 out of 10 mice still maintaining B cell aplasia in the nonirradiated m19-humBBz group (Figure 3C). In contrast, irradiation did not significantly impact B cell killing by m1928z CAR T cells in the blood or BM (Figure 3C).

With evidence of enhanced in vivo persistence and previous studies demonstrating that 4-1BB costimulation is essential for T cell survival and antiapoptosis (35) we evaluated CAR T cell viability and proliferation. From multiple independent productions, m19-humBBz CAR T cells showed a slightly increased, although not significantly higher viability and proliferation than m1928z CAR T cells (Figure 4, A and B). We also evaluated the expression of antiapoptotic proteins, BCL2 and BCL-XL, by flow cytometry. 
A

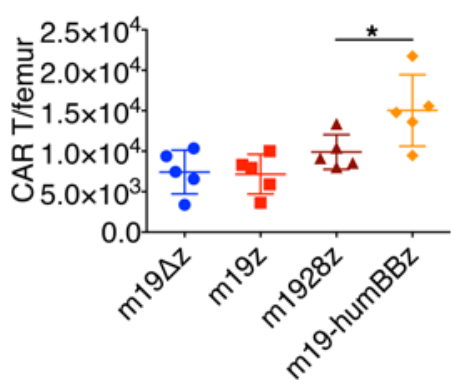

B

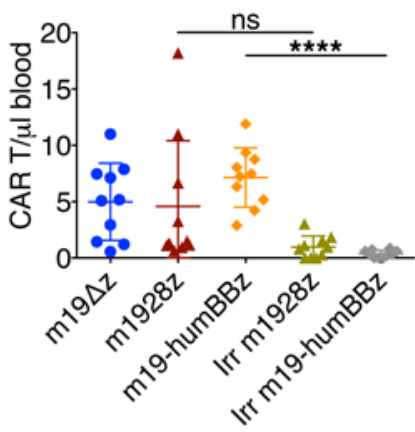

C

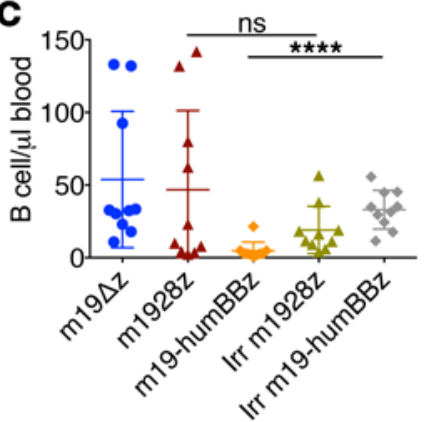

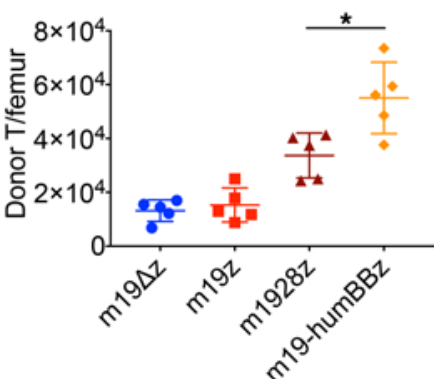
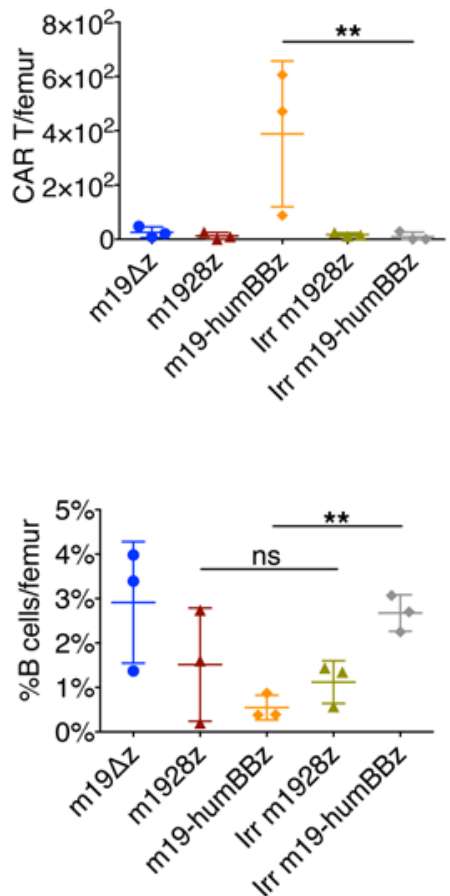

Figure 3. Persistence of m19-humBBz CAR $T$ cells is required for optimal function in vivo. (A) CAR T (CD3+CAR $)$ and donor $\mathrm{T}\left(\mathrm{CD}^{+}\right.$Thy $\left.1.1^{+}\right)$cell numbers in Rag $1^{-1-}$ mice 1 week after transfer. One million CAR T cells were i.v. injected into Rag1 ${ }^{-1-}$ mice. One week later, bone marrow (BM) cells were isolated, stained, and analyzed by flow cytometry. Each data point indicates 1 mouse ( $n=5$ per group). (B and C) One million irradiated or nonirradiated CAR T cells were i.v. injected into cyclophosphamide-preconditioned ( $300 \mathrm{mg} / \mathrm{kg}$ ) C57BL/6 mice. One week later, blood and BM were collected, stained, and analyzed by flow cytometry. (B) Irradiated and nonirradiated CAR T cell (CD3+CAR ${ }^{+}$numbers in the blood and BM 1 week after transfer. (C) B cell $\left(B 220^{+} \mathrm{CD} 19^{+}\right)$numbers in the blood and B cell percentages in the BM 1 week after CAR T transfer. Data are from 1 experiment. Each data point indicates 1 mouse. $n=10$ per group for blood samples; $n=3$ per group for BM samples. ${ }^{*} P<0.05$; ${ }^{* *} P<0.01 ;{ }^{* * *} P<0.0001$ by unpaired $t$ test. ns, not significant.

Without antigen stimulation, m19-humBBz CAR T cells have 1.6-fold higher BCL2 (mean fluorescence intensity [MFI] 1,107 vs. 668) and 1.9-fold higher BCL-XL (MFI 6,185 vs. 3,305) than m1928z CAR T cells (Figure 4C). We also evaluated the expression of antiapoptotic proteins by Western blotting after antigen stimulation. The m19-humBBz CAR T cells have greater BCL2 (3.6 vs. 1.8) and BCL-XL (5.7 vs. 0.01) expression than $\mathrm{m} 1928 \mathrm{z}$ CAR $\mathrm{T}$ cells after normalization to $\beta$-actin (Figure $4 \mathrm{D}$ ).

4-1BB costimulation induces greater $N F-\kappa B$ than $C D 28$ costimulation in mouse CAR T cells. We sought to identify signaling pathways that regulate in vivo persistence in CAR $T$ cells to optimize these pathways and further enhance persistence since loss of CAR T cells led to relapses in patients (15). Therefore, we sorted $\mathrm{m} 19 \mathrm{z}$, m19-humBBz, and m1928z CAR T cells after antigen stimulation and performed RNA sequencing (RNASeq), which confirmed that each CAR group had a unique transcriptional profile (Supplemental Figure 6, A and B). Gene set enrichment analysis (GSEA) revealed enrichment for pathways that regulate NF- $\mathrm{kB}$ when comparing CAR T cells that costimulate 4-1BB versus CD28 or lack costimulation (Supplemental Figure 6C). $\mathrm{NF}-\mathrm{kB}$ is a key regulator of $\mathrm{T}$ cell survival (36) as well as antitumor control (37), so we evaluated if differential 
A

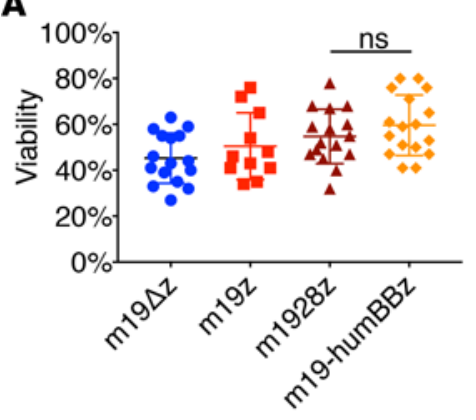

B

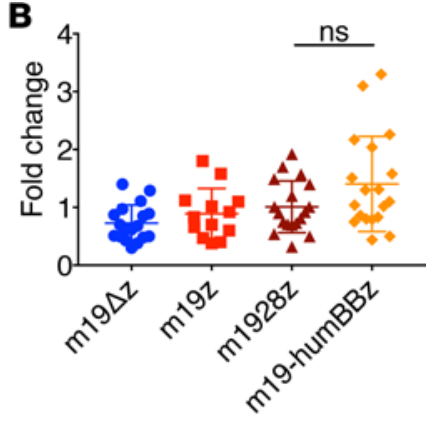

C

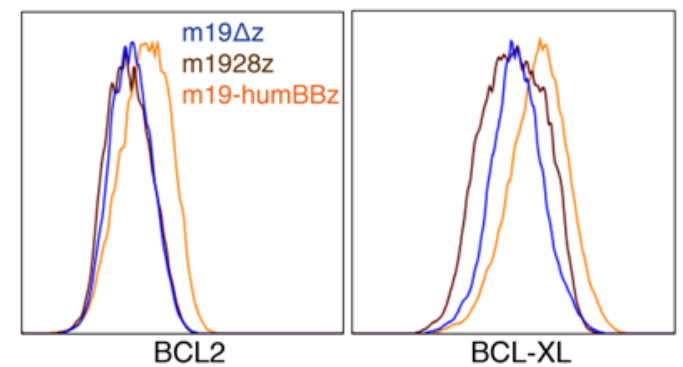

D

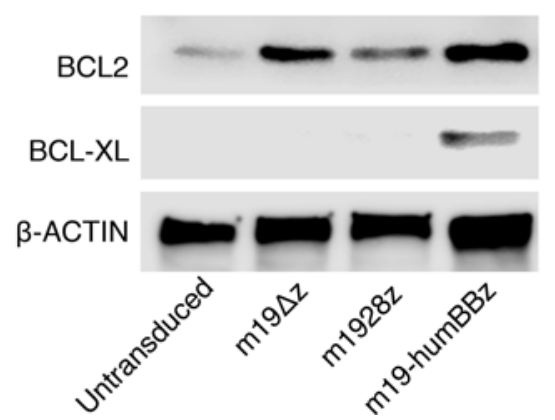

Figure 4. $m$ 19-humBBz CAR $T$ cells have higher antiapoptotic protein expression than m1928z CAR $T$ cells. Viability (A) and proliferation (B) of mCD19-targeted CAR T cells. CAR T cells were produced and proliferation was evaluated by fold change from the initial cell number to final cell yield at day 4. Cell viability was measured by trypan blue staining on an automated cell counter (Bio-Rad). Data were pooled from 17 (viability) and 19 (proliferation) independent productions. (C) BCL2 and BCL-XL expression in CAR T cells by flow cytometry. Day 4 CAR T cells were intracellularly stained with anti-BCL2 and anti-BCL-XL antibodies and analyzed by flow cytometry. Cells were pregated on live CAR T cells. Data are representative of 2 independent experiments. (D) BCL2 and BCL-XL protein expression after antigen stimulation. One million day 4 CAR T cells were stimulated on $1 \times 10^{5} 3$ T3-mCD19 cells for 4 hours. Cell lysates from CAR T cells were prepared, protein quantified by BCA, normalized to total protein, and analyzed by Western blot. Western blots are representative of 2 independent experiments. Semiquantitation of Western blots was done using ImageJ software. BCL2 and BCL-XL expression in different CAR T cells was compared by normalizing to $\beta$-actin. Data were analyzed by unpaired $t$ test. ns, not significant.

levels of NF-kB account for enhanced in vivo CAR T cell persistence and/or function of m19-humBBz CAR T cells. Mechanistic studies (30) of 4-1BB costimulation have been performed with 293 cells transduced with wild-type 4-1BB. We utilized a similar reporter cell line, NF-kB/293/GFP-Luc, which allows measurement of GFP fluorescence as an indicator of NF- $\mathrm{KB}$ signaling. $\mathrm{mCD} 19$-targeted CARs were retrovirally transduced into NF-kB/293/GFP-Luc reporter cells and only m19-humBBz transduction induced NF-кB (Figure 5A). We validated this observation in primary $\mathrm{mCD} 19$-targeted CAR T cells stimulated with antigen. CAR T cells were produced from NF-kB-RE-luc transgenic mice, which have a firefly luciferase transgene regulated by NF-kB-responsive elements (38). After 4-hour stimulation with 3T3-mCD19 AAPCs, CAR T cell lysates were prepared and evaluated for bioluminescence. Compared with $\mathrm{m} 19 \Delta \mathrm{z}, \mathrm{NF}-\mathrm{kB}$ signaling increased by 29-fold in m19-humBBz CAR T cells and approximately 5-fold in m1928z CAR T cells (Figure 5B).

Mutations of the 4-1BB costimulatory domain modulate NF- $\kappa B$ and in vitro function of human CD19-targeted CAR T cells. We demonstrated that mCD19-targeted $\mathrm{T}$ cells with a CAR containing a 4-1BB domain have enhanced proliferation and NF- $\mathrm{KB}$ signaling. We wanted to validate these observations in primary human $\mathrm{T}$ 
A

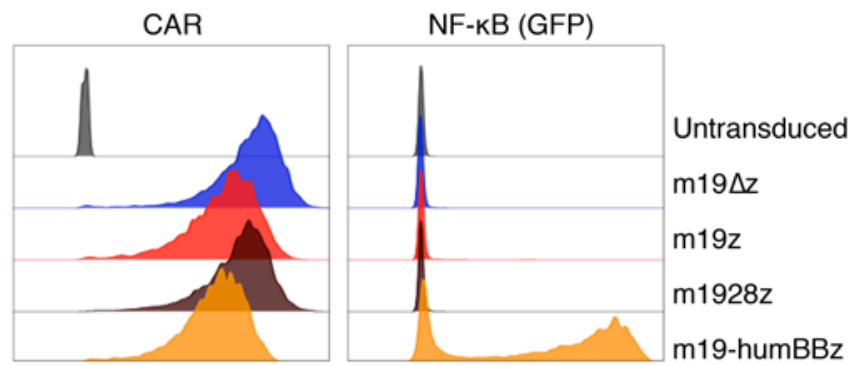

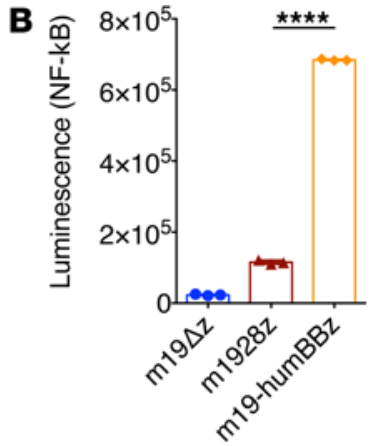

C
D

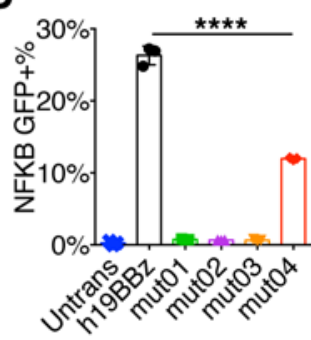

E

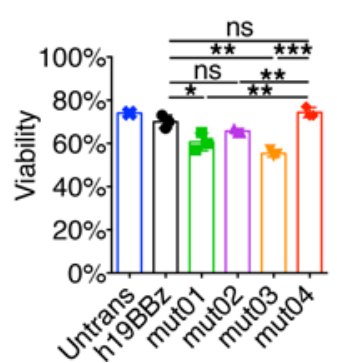

$\mathbf{F}$

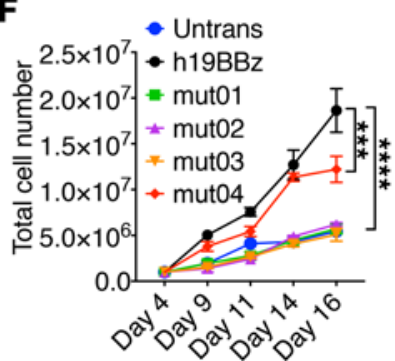

G

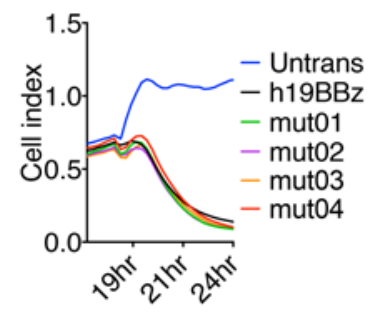

Figure 5. NF-KB signaling regulates the viability and proliferation of 4-1BB-based CAR T cells. (A) CAR expression (mCherry) after transduction (left) and NF- $\kappa B$ upregulation (right) in NF- $\kappa B / 293 /$ GFP-Luc reporter cells. Reporter cells were transduced with mouse CD19-targeted CARs and NF- $\kappa B$ signaling was measured by flow cytometry for GFP. Data are representative of 2 independent experiments. (B) m19-humBBz CAR T cells have greater NF- $\kappa B$ signaling than m1928z CAR T cells after antigen stimulation. Three million CAR T cells derived from NF-kB-RE-luc transgenic mice were cocultured with $3 T 3-m C D 19$ cells at a 10:1 ratio for 4 hours. Cell lysates were evaluated using a luciferase assay. Data are representative of 3 independent experiments in triplicate. (C) Amino acid sequences of human wild-type and mutated 4-1BB endodomains evaluated in hCD19-targeted CARs. Putative TRAF binding domains or mutations are in red. Amino acid numbers of the 4-1BB endodomain are shown. (D) NF- $\kappa B$ signaling of hCD19 CAR-transduced reporter cells. NF- $\kappa B / 293 / G F P-L u c$ reporter cells were retrovirally transduced with hCD19-targeted CARs. Percentages of GFP+ cells, which reflect NF- $\mathrm{kB}$ signaling, were measured by flow cytometry. Data are from 1 experiment and done in triplicate. (E) Viability on day 16 and (F) proliferation of hCD19-targeted CAR T cells cultured in vitro. Human T cells were isolated from healthy donor peripheral blood mononuclear cells at day 0. CAR T cells were harvested, beads removed and cocultured with 3T3-hCD19 cells at a 5:1 ratio for 2 weeks. Cell numbers were measured at indicated time points. (C) Cytotoxicity of hCD19-targeted CAR T cells. CAR T cells were cocultured with 3T3-hCD19 cells at a 10:1 ratio. Target cell killing was monitored by XCELLigence RTCA (real-time cell analysis). For E, F, and G, data are from a single experiment in triplicate. Data in $\mathbf{B}-\mathbf{F}$ represent the mean $\pm \mathrm{SD}$. Cytotoxicity curves show the mean only. ${ }^{*} P<0.05 ;{ }^{* *} P<0.01$; ${ }^{* * *} P<0.001 ;{ }^{* * *} P<$ 0.0001 by unpaired $t$ test (B, D, and $\mathbf{E}$ ) or 2-way ANOVA (F). ns, not significant.

cells and extend them by directly evaluating if NF-kB signaling correlated with CAR T cell viability and proliferation. We developed human CD19-targeted (hCD19-targeted) CARs (Figure 5C) containing a wild-type (h19BBz) or mutated 4-1BB endodomain (mut01-mut04) to modulate NF- $\mathrm{kB}$ signaling. The 4-1BB endodomain mutants were located in previously identified $(29,30,32,33)$ TRAF1-3 binding domains. We measured the ability of the hCD19-targeted CARs to induce NF-kB in reporter cells. The h19BBz CAR and one with double 4-1BB endodomains (mut04) had high (26\%) and moderate (12\%) levels of NF-kB upregulation, respectively (Figure 5D). However, all 3 CARs with mutated TRAF binding domains (mut01-03) showed minimal NF- $\mathrm{kB}$ induction after transduction (Figure 5D). Next, we evaluated how these differential levels of $\mathrm{NF}-\mathrm{kB}$ signaling impact in vitro function of human $\mathrm{T}$ cells. We retrovirally transduced healthy donor human T cells with the h19BBz or mutated CARs, which displayed similar gene transfer and CD4/CD8 ratios (Supplemental Figure 7). Cell growth was monitored after stimulation with 3T3-hCD19 AAPCs. Human CAR T cells with greater levels of NF-кB signaling (h19BBz and mut04) also had the greatest viability $(70 \%-74.3 \%)$ 
A
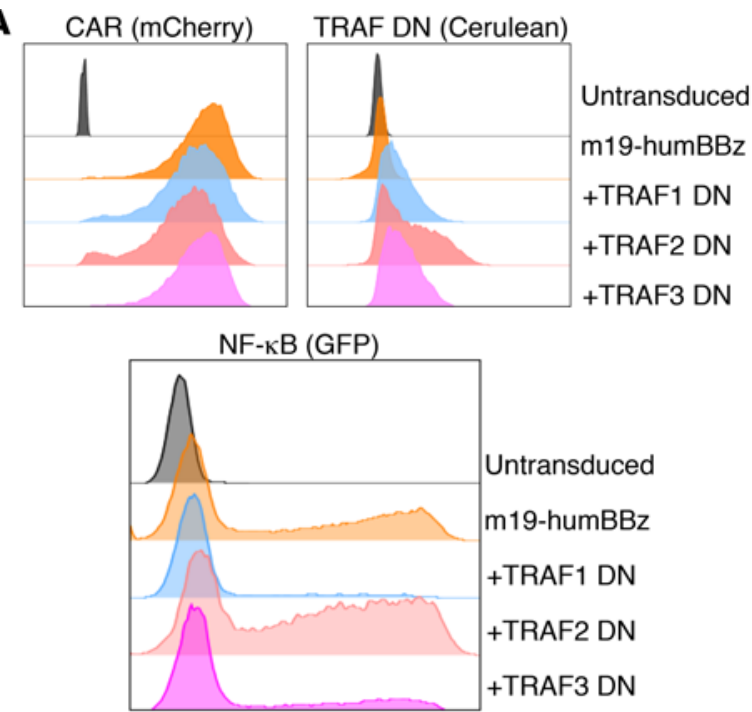

B
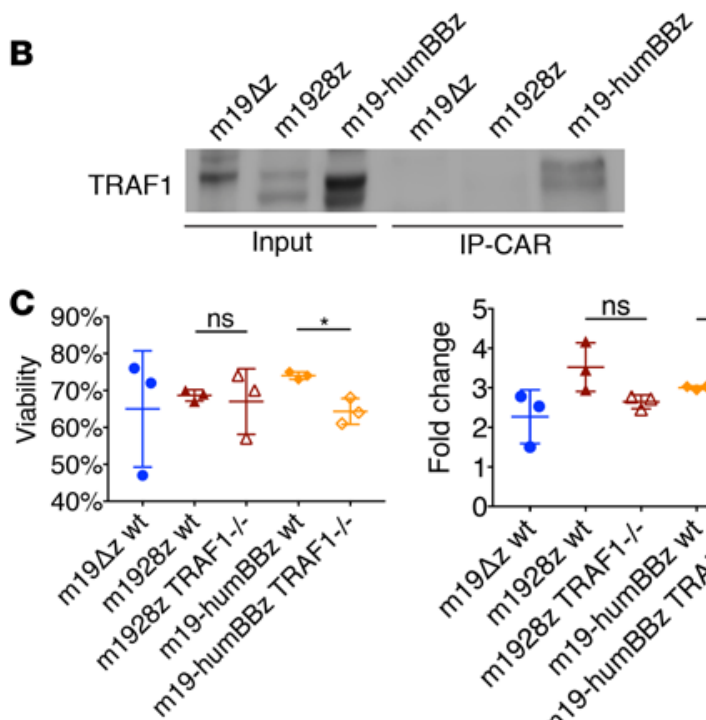

D

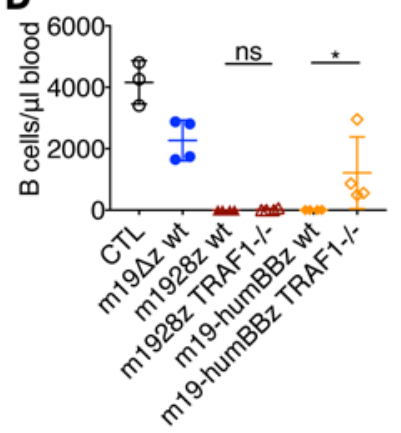

Figure 6. TRAF1 inhibition negatively impacts NF-KB signaling and m19-humBBz CAR T cell function. (A) Effect of TRAF dominant-negative (DN) proteins on m19-humBBz-induced NF- $\kappa B$ signaling in NF-KB/293/GFP-Luc reporter cells. Reporter cells were retrovirally transduced with cerulean-tagged TRAF1 DN, TRAF2 DN, or TRAF3 DN constructs followed by transduction with m19-humBBz CAR. Cells were analyzed by flow cytometry for NF- $\mathrm{kB}$ signaling, shown as $\mathrm{GFP}^{+}$cells. Data are representative of 2 independent experiments. (B) Western blot of NF- $\kappa B / 293$ cell lysates before and after CAR immunoprecipitation. Cell lysates were prepared from CAR-transduced NF-KB/293 cells, ligated to Protein $L$ magnetic beads, enriched with a magnet, electrophoresed, and probed for TRAF1. Data are from 1 single experiment. (C) Viability and proliferation of wild-type and Traf1 $^{-1-}$ mCD19-targeted CAR T cells. CAR T cells were produced from wild-type B6 mice or Traf1 $1^{-1-}$ mice and proliferation was evaluated by fold change from the initial cell number to final cell yield at day 4 . Cell viability was measured by trypan blue staining on an automated cell counter (Bio-Rad). Data are from a single experiment in triplicate. (D) In vivo B cell killing and CAR T persistence in the blood 2 weeks after CAR T transfer. CAR T cells prepared from wild-type B6 or Traf1 $1^{-1-}$ mice were adoptively transferred at $3 \times 10^{5}$ dose into cyclophosphamide-preconditioned Thy1.1 mice. Blood was collected for flow cytometry. Counting beads were used to quantify cell numbers. Each data point indicates 1 mouse ( $n=4$ per group). ${ }^{*} P<0.05 ;{ }^{* *} P<0.001$ by unpaired $t$ test. ns, not significant.

compared with CAR T cells with mutations (mut01-mut03, 55.3\%-65.6\%) in the TRAF binding domains (Figure 5E). Similarly, both h19BBz and mut04 hCD19-targeted CAR T cells proliferated to a significantly greater extent (18.6- and 12.2-fold, respectively) than mut01-mut03 hCD19-targteted CAR T cells (approximately 5-fold, Figure 5F). However, at an E/T ratio of 10:1 all groups of CAR T cells killed 3T3-hCD19 cells similarly, despite differences in NF- $\mathrm{kB}$ signaling (Figure $5 \mathrm{G}$ ).

TRAF1/NF- $K B$ is required for optimal m19-humBBz CAR T cell function in vivo. Studies have demonstrated that NF- $\mathrm{KB}$ signaling in T cells is mediated, at least in part, through the binding of TRAF1-3 to 
A

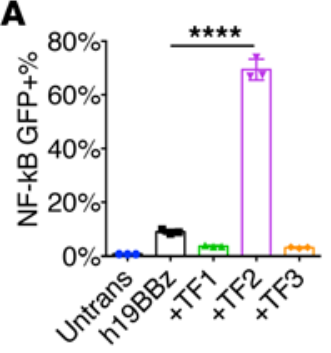

E

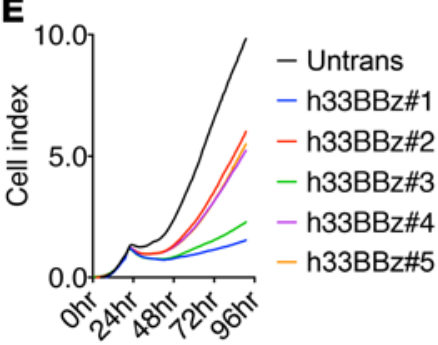

B

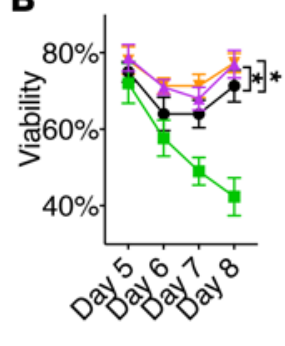

$\mathbf{F}$

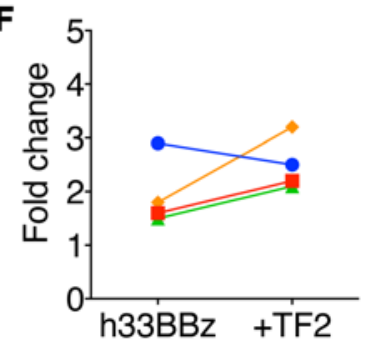

C

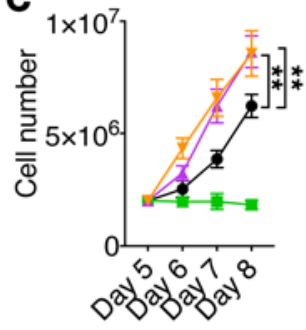

D

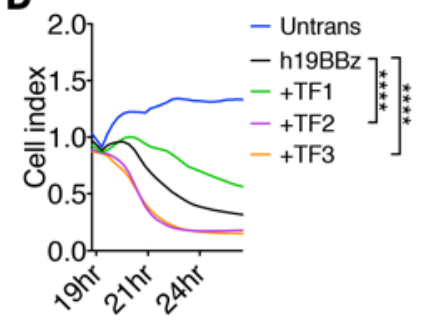

G

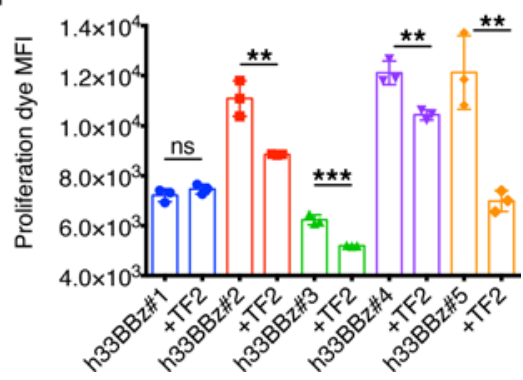

Figure 7. TRAF2 overexpression modulates 4-1BB-based human CAR T function. (A) NF- $\kappa B$ signaling in human CD19 CAR-transduced (h19BBztransduced) NF- $\mathrm{B} / 293 /$ GFP-Luc reporter cells by increasing NF- $\kappa B$. Cells were transduced with h19BBz CAR with or without TRAFs. NF- $\mathrm{BB}$ was measured by GFP fluorescence. Data are from 1 experiment in triplicate. (B) Viability and (C) cell expansion of h19BBz CAR T cells with TRAF overexpression upon antigen stimulation. CAR T cells were cocultured with 3T3-hCD19 at a 10:1 ratio and cell numbers and viability were measured daily for 3 days. (D) Cytotoxicity of h19BBz CAR T cells with TRAF overexpression. CAR T cells were cocultured with 3T3-hCD19 at a 5:1 ratio. Target cell killing was monitored by xCELLigence RTCA (real-time cell analysis) system. (E) Cytotoxicity of human CD33-targeted CAR (h33BBz) T cells with different scFvs. CAR T cells were cocultured with CHO-hCD33 at a 10:1 ratio. Target cell killing was monitored by RTCA. (F) Fold change of h33BBz T cell production with or without TRAF2 cotransduction. CAR T cells were produced and proliferation was evaluated by fold change from the initial cell number to final cell yield. (G) h33BBz CAR T cell expansion in vitro upon antigen stimulation. CAR T cells were stained with proliferation dye and cocultured with CHO-hCD33 at a 10:1 E/T ratio for 4 days. Proliferation of CAR T cells was evaluated by flow cytometry (MFI of proliferation dye in CAR T population). All experiments other than $\mathbf{F}$ were done in triplicate. For $\mathbf{B}, \mathbf{C}$, and $\mathbf{D}$, data are 1 representative of 3 donors. For $\mathbf{F}$ and $\mathbf{G}$, data are from 1 single experiment. For $\mathbf{A}$ and $\mathbf{E}$, data are 1 representative of 2 independent experiments. Cytotoxicity data are shown as the mean only; others are shown as the mean \pm SD. Untrans, untransduced; +TF1, CAR plus TRAF1; +TF2, CAR plus TRAF2; +TF3, CAR plus TRAF3. ${ }^{*} P<0.05 ;{ }^{* *} P<0.01 ;{ }^{* * *} P<0.001 ;{ }^{* * * * P}$ $<0.0001$ by unpaired $t$ test ( $\mathbf{A}$ and $\mathbf{C}), 2$-way ANOVA (B and $\mathbf{C}$ ), or Kolmogorov-Smirnov test ( $\mathbf{D}$ and $\mathbf{E})$. ns, not significant.

the intracellular domain of 4-1BB (29-33). It is speculated that TRAF2 is critical for enhanced function mediated by 4-1BB costimulation in CAR T cells, but no direct evidence exists $(27,39)$. Furthermore, our analyses of the function of human $\mathrm{T}$ cells modified with CARs containing mutated 4-1BB (Figure $5 \mathrm{D})$ demonstrate that NF- $\mathrm{kB}$ signaling correlates with hCD19-targeted CAR T cell proliferation and viability but can not distinguish which TRAFs are modulating NF-KB since the targeted domains can bind TRAF1, -2, or -3. Therefore, we applied our models to determine if TRAF1, TRAF2, or TRAF3 regulated NF-KB signaling and CAR $\mathrm{T}$ cell function.

We introduced TRAF dominant-negative (DN) proteins into NF-kB/293/GFP-Luc reporter cells followed by m19-humBBz CAR transduction. Gene transfer for CAR and TRAF DN proteins was confirmed by flow cytometry (Figure 6A). Compared with cells transduced with only m19-humBBz, NF-kB signaling with TRAF1 DN decreased (Figure 6A, GFP\%: $45.5 \%$ vs. $13.4 \%$; GFP MFI: 10,929 vs. 1,688 ). The TRAF3 DN group also displayed decreased NF-kB (Figure 6A, GFP $\%$ : $45.5 \%$ vs. $30.8 \%$; GFP MFI: $10,929$ vs. 6,056$)$, although not to the same extent as the TRAF1 DN group. In contrast, NF-kB signaling in the TRAF2 DN group was greater than the m19-humBBz control (Figure $6 \mathrm{~A}, \mathrm{GFP}^{+} \%: 63.5 \%$ vs. $45.5 \%$; GFP MFI: 14,309 vs. 10,929). We also evaluated if TRAF1 could be identified binding to the CAR. After transduction of NF-kB/293/GFP-Luc reporter cells with the m19-humBBz CAR we isolated the CAR by Protein $L$ binding and assayed for retention of TRAF1. We detected TRAF1 in both the total protein lysate as well as the immunoprecipitate, confirming that TRAF1 binds to the m19-humBBz CAR (Figure 6B).

We aimed to validate that TRAF1 was required for 4-1BB costimulatory enhancement of mCD19-targeted CAR T cell function by evaluating CAR T cells, derived from wild-type C57BL/6 mice or Traft-1(40) mice, after adoptive transfer into immune-competent mice. For immune phenotype, both m1928z and m19-humBBz Traf1 ${ }^{--}$CAR $T$ cells have a higher CD4/CD8 ratio and a greater frequency of central memory 
cells $\left(\mathrm{CD}_{2} \mathrm{~L}^{+} \mathrm{CD} 44^{+}\right)$(Supplemental Figure 8). Also, m19-humBBz Traf1 ${ }^{-1-}$ CAR T cells had significantly lower viability and proliferation than $\mathrm{m} 19$-humBBz wild-type CAR $\mathrm{T}$ cells, but $\mathrm{m} 1928 \mathrm{z}$ CAR $\mathrm{T}$ cell viability or proliferation was not significantly affected by lack of TRAF1 (Figure 6C). In vivo, B cells recovered 2 weeks after treatment with m19-humBBz Traf1 ${ }^{-/}$CAR T cells, while m19-humBBz wild-type CAR T cells maintained B cell aplasia (Figure 6D). Correspondingly, CAR T cell persistence in the m19-humBBz Traf1 $1^{-1}$ group was significantly decreased compared with the $\mathrm{m} 19$-humBBz wild-type CAR $\mathrm{T}$ cell group (Figure 6D). However, the persistence of m1928z CAR T cells, or B cell killing, was not significantly reduced when the donor T cells were TRAF1 deficient (Figure 6D).

Increasing $N F-\kappa B$ signaling enhances $C A R T$ cell function. We hypothesize that the reduced efficacy of m19-musBBz CAR T cells is due to suboptimal NF- $\kappa B$ signaling and can be optimized by mutations that enhance NF- $\kappa B$ signaling. Therefore, we created a m19-musBBz mut01 CAR that substituted the first 5 $\mathrm{N}$-terminal amino acid mismatches (underlined in Figure 2A) of mouse 4-1BB with human 4-1BB amino acids. This region of human $4-1 \mathrm{BB}$ has been previously identified to bind TRAF3 to a greater extent than its mouse counterpart (30), which we characterized as being required for optimal NF- $\mathrm{B}$ signaling (Figure

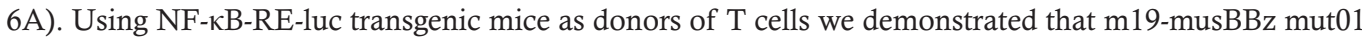
CAR T cells have approximately 2-fold greater NF- $\mathrm{kB}$ signaling compared with $\mathrm{m} 19-\mathrm{musBBz}$, which correlated with increased cytokine production and antiapoptotic protein production (Supplemental Figure 9 , $\mathrm{A}-\mathrm{C}$ ). In vivo evaluation of $\mathrm{m} 19-\mathrm{musBBz}$ mut01 demonstrated B cell killing and CAR T cell persistence similar to m19-humBBz and significantly greater than m19-musBBz (Supplemental Figure 9, D-G).

While mutating the 4-1BB costimulatory domain to increase TRAF binding and NF- $\mathrm{kB}$ signaling is one strategy to enhance CAR $\mathrm{T}$ cell function, we aimed to demonstrate that another is to provide excess TRAF proteins in CAR T cells that utilize 4-1BB costimulation. We cotransduced the h19BBz CARs and TRAFs in the NF- $\mathrm{BB} / 293 / \mathrm{GFP}-L$ uc reporter cells. Compared with reporter cells transduced with the h19BBz CAR alone, TRAF2 supported a dramatic increase in NF-kB, while excess TRAF1 or TRAF3 had negligible effects (Figure 7A). We also cotransduced primary human T cells with h19BBz and TRAFs to evaluate the impact on CAR T cell function. Both TRAF2 and TRAF3 transduction significantly increased viability, proliferation, and target killing of h19BBz CAR T cells (Figure 7, B-D). However, cotransduction of TRAF1 with h19BBz CAR T cells resulted in decreased viability, proliferation, and target killing of h19BBz CAR T cells, which may be due to reduced CAR expression (Supplemental Figure 10, A and B). Increased TRAF expression also modulates cytokine production after antigen stimulation (Supplemental Figure 10C). We also compared the in vivo activity of NSG mice treated with the NALM6 leukemia cell line followed by the adoptive transfer of h19BBz CAR T cells with or without excess TRAF2 (Supplemental Figure 11). Both h19BBz CAR T cell groups enhanced leukemia killing and survival compared with untransduced $\mathrm{T}$ cells, but neither group appeared superior to each other. This may be due to the nature of the aggressiveness of the NALM6/NSG mouse model (27), which requires rapid leukemia killing so that even second-generation CAR T cells do not prevent death.

We have demonstrated the role of NF-kB and TRAFs in regulation of both human and mouse CD19targeted CAR T cell function; however, we can not exclude that the CD19 antigen may contribute to these results. Therefore, we evaluated how proliferation of CD33-targeted CAR T cells was impacted by overexpression of TRAF2, which greatly increases NF-кB (Figure 7A). We created 5 de novo CD33-targeted CARs with the same design of the h19BBz CAR but replacing the anti-CD19 scFv with an anti-CD33 scFv and followed by the CD8 hinge and transmembrane domain, 4-1BB costimulatory domain, and $\mathrm{CD} 3 \zeta$. We validated the function of our CD33-targeted human CAR T cells by demonstrating cytotoxicity of CD33-postive targets in vitro (Figure 7E). Overexpression of TRAF2 enhanced the number of CAR T cells produced, as well as their proliferation after antigen simulation, in 4 of the 5 CD33-targeted CAR T cells assayed (Figure 7, F and G).

\section{Discussion}

In our immune-competent animal model, high doses of m19-musBBz CAR T cells had anti-leukemia efficacy equivalent to that of m1928z CAR T cells; however, at a stress-test dose $\left(3 \times 10^{5}\right)$ they had reduced efficacy (Figure 1, C-F). We hypothesized that the m19-musBBz CAR was suboptimal and sequence modifications could increase its efficacy to be equivalent to the m1928z CAR. Therefore, we replaced the mouse 4-1BB endodomain with the human 4-1BB endodomain (m19-humBBz) (Figure 2). The in vivo function (Figure 2D) of m19-humBBz CAR T cells was equivalent to m1928z CAR T cells at stress-test dose levels, 
which is consistent with clinical results that demonstrate equivalent complete response rates in patients treated with h19BBz or h1928z CAR T cells $(16,21,22,41)$. However, the in vitro function of m19-humBBz CAR T cells, as measured by cytokine production and cytotoxicity, was inferior compared with $\mathrm{m} 1928 \mathrm{z}$ CAR T cells (Figure 2, B and C). These results are consistent with prior studies that demonstrated human CAR T cells provided CD28 costimulation-secreted cytokines, such as IL- 2 , IFN- $\gamma$, and TNF- $\alpha$, at greater levels than CARs with 4-1BB costimulatory domains $(11,13,42,43)$.

Efficacious in vivo leukemia eradication despite inferior in vitro function appeared inconsistent with an optimal cytotoxic CAR T cell, so we evaluated potential mechanisms that could compensate. Others (27) have observed that increased persistence of h19BBz CAR T cells supported enhanced malignant B cell killing in immune-deficient mice, but the tumor killing was not equivalent to h1928z CAR T cells and all the mice in this study died rapidly from leukemia progression regardless of CAR evaluated. We considered that antigen may be a confounding variable between the previous study and ours (Figures 1 and 2), in light of a recent study that demonstrated CAR T cell exhaustion could be induced upon engagement of antigen

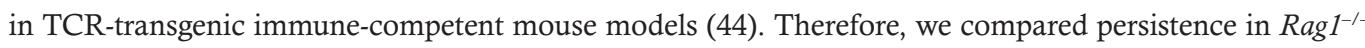
mice and determined that mouse $\mathrm{T}$ cells modified with a CAR containing the 4-1BB costimulatory domain supported the greatest persistence (Figure 3A). Furthermore, when we irradiated mCD19-targeted CAR $\mathrm{T}$ cells before infusion into immune-competent mice only m19-humBBz CAR T cells had significantly reduced persistence and B cell killing, while m1928z CAR T cell persistence and B cell killing was not significantly affected, demonstrating that persistence is critical for enhancement of in vivo CAR T cell function mediated by 4-1BB costimulation (Figure 3).

Additional support of the enhanced persistence of CAR T cells with 4-1BB costimulation is the increase of antiapoptotic proteins in m19-humBBz CAR T cells, which we believe is a novel observation (Figure 4). We compared gene expression of the $\mathrm{mCD} 19$-targeted CAR $\mathrm{T}$ cell groups to identify pathways that could contribute to enhanced antiapoptosis and/or persistence imparted by 4-1BB costimulation. GSEA demonstrated that m19-humBBz differed in expression of an NF- $\mathrm{kB}$ regulatory pathway when compared with $\mathrm{m} 19 \mathrm{z}$ or m1928z CAR T cells, which was similar to enrichment of NF-kB regulatory genes in m19-musBBz (Supplemental Tables 1-4 and Supplemental Figure 6). Using a 293 reporter cell line we identified $N F-\kappa B$ signaling only with the m19-humBBz CAR and also validated that NF- $\kappa B$ signaling is enhanced in m19-humBBz mouse CAR T cells compared with m1928z mouse CAR T cells (Figure 5, A and B). While $\mathrm{NF}-\kappa \mathrm{B}$ is known to be critical for $\mathrm{T}$ cell function $(36,37)$, we determined that the level of NF- $\mathrm{kB}$ signaling for CARs with a 4-1BB costimulatory domain is much greater than CARs with a CD28 costimulatory domain, which likely contributes to the differential in vivo function of CD19-targeted CAR T cells. We also validated our observation in primary human CAR T cells. Mutations of 4-1BB in an anti-human CD19 CAR variably reduced NF- $\mathrm{kB}$, which correlated with the attenuation of viability and/or proliferation (Figure 5, C-F).

Recent studies suggest that CD28 costimulation directs differentiation of human CAR T cells to effector memory, while 4-1BB costimulation promotes differentiation to central memory cells $(27,45,46)$. Furthermore, these studies identified distinct metabolic and gene expression pathways associated with the CD28 or 4-1BB endodomains and they suggest that 4-1BB costimulation promotes CAR T cell persistence and protects against CAR T cell exhaustion $(27,45,46)$. Therefore, we can not rule out that these distinct metabolic or gene expression patterns after costimulation dictate the role of NF- $\mathrm{KB}$ signaling in CAR T cells. However, it may also be that NF- $\mathrm{KB}$ signaling drives different metabolic or signaling pathways in CAR T cells, which is a hypothesis that we are evaluating. In fact, prior studies have established that NF- $\mathrm{\kappa B}$ signaling is required for maintaining memory $\mathrm{T}$ cells and can also increase mitochondrial respiration $(47,48)$.

The critical role for $\mathrm{NF}-\kappa \mathrm{B}$ in 4-1BB costimulatory enhancement of CAR $\mathrm{T}$ cell function suggests a mechanism for the reduced efficacy of the m19-musBBz CAR. After antigen stimulation, NF- $\mathrm{kB}$ signaling of m19-humBBz CAR T cells is 4.7 times greater than m19-musBBz CAR T cells (Supplemental Figure 9A). There are a total of 19 amino acid differences between the mouse and human 4-1BB endodomains and only one of them is located in the QEE domains identified critical for costimulation, and the $\mathrm{Q} \rightarrow \mathrm{E}$ substitution is reported to not affect $4-1 \mathrm{BB}$ costimulation $(29,30)$. By mutating 5 amino acids in the N-terminal portion of the mouse 4-1BB endodomain we improved NF- $\mathrm{BB}$ signaling in mCD19-targeted CAR T cells, which resulted in enhanced cytokine production and antiapoptotic protein expression, as well as in vivo B cell killing and CAR T persistence (Supplemental Figure 9, E-G). Arch and Thompson (30) mutated this same domain in mouse 4-1BB and reported that TRAF3 recruitment was enhanced. This suggests that optimization of TRAF recruitment to costimulatory domains can enhance CAR $T$ cell function. TRAF proteins 
regulate $\mathrm{T}$ cell function by linking extracellular activation receptors, including 4-1BB, and intracellular signaling pathways, thereby impacting $\mathrm{T}$ cell differentiation, proliferation, survival, and cytokine production $(36,49)$. TRAF proteins have been speculated as having multiple roles in transducing 4-1BB costimulation in CARs, but to date none have been able to confirm or define their specific roles $(27,39)$. Using a 293 reporter we determined that TRAF1 and TRAF3 are required for optimal NF- $\mathrm{kB}$ activation by $4-1 \mathrm{BB}$ costimulation, which we confirmed for TRAF1 in primary mouse CAR T cells (Figure 6). A TRAF2-DN inhibitor increased NF- $\mathrm{kB}$ signaling of m19-humBBz CARs, which may be due to its role in degrading the NF- $\mathrm{kB}-$ inducing kinase (NIK) since it serves as a negative regulator of the alternative NF- $\kappa B$ signaling pathway (25). While depletion of TRAFs negatively impacted CAR T cell function, overexpressing TRAF2 or TRAF3 in primary human h19BBz CAR T cells enhanced viability, proliferation, and cytotoxicity (Figure 7, A-D). T cells with h19BBz and excess TRAF2 dramatically increased NF- $\mathrm{B}$ compared with h19BBz CAR T cells (7.7-fold), further confirming that increased NF- $\mathrm{BB}$ enhances CAR T cell function (Figure 7A). However, we also observed enhancement of CAR T cell function in some groups that is independent of NF- $\kappa$ B. Human $\mathrm{T}$ cells with the h19BBz CAR and excess TRAF3, despite having enhanced function, had negligible changes in NF- $\mathrm{kB}$, which suggests TRAF3 may be mediating its potentiating effects through another signaling pathway(s). We suspect this may be through enhancement of endogenous CD28 costimulation since TRAF3 is required for TCR/CD28 signaling (50). We also validated our observation for the role of excess TRAF2 in enhancing CAR T cell proliferation with CD33-targeted CAR T cells (Figure 7, E-G).

Transduction of TRAFs and CARs into T cells may allow potentiation of both 4-1BB and CD28 costimulation. Combining both TRAFs and CARs may substitute for a third-generation CAR design that includes both CD28 and 4-1BB endodomains, which were envisioned with the goal of enhancing both cytotoxicity and persistence $(43,51)$. However, both these designs may be hampered by trying to merge phenotypes that are mutually exclusive, although dissociation of the costimulatory domains has had some success with enhancing third-generation CAR T cell function (27). Furthermore, continuous TRAF enhancement of $4-1 \mathrm{BB}$ costimulation and NF- $\mathrm{\kappa B}$ signaling could result in tonic signaling and CAR T cell death (39) or even carcinogenesis (52), suggesting that an optimal TRAF-plus-CAR design may require a molecular switch to regulate TRAF expression.

The clinical evaluation of hCD19-targeted CAR T cells in patients has generated promising results, which is represented by the recent approval of 3 CAR therapies for B cell malignancies. Understanding the biology of this unique cellular immunotherapy will be important to improve efficacy and reduce toxicity. Our study demonstrates that enhancement of CAR T function by 4-1BB requires TRAF1 and TRAF3 to optimally activate NF-kB. Furthermore, our strategy of coexpressing a 4-1BB-based CAR and TRAF proteins enhanced CAR $\mathrm{T}$ cell viability, proliferation, and cytotoxicity. Overexpressing TRAF proteins could also benefit CD28-based CAR T cells since some TRAFs interact with CD28 (50). Considering the antagonistic roles of both TRAF1 and TRAF2 in the NF- $\kappa B$ pathway and for the role of TRAF3 in both $4-1 \mathrm{BB}$ and CD28 costimulation it will be necessary to evaluate the impact of individual TRAFs in CARs with different costimulatory domains to identify how they regulate optimal CAR T cell signaling and function.

\section{Methods}

Mice. C57BL/6, Thy1.1 (B6.PL-Thy1 ${ }^{a}$ CyJ), and Rag1-/- (B6.129S7-Rag1 $1^{\text {tmIMom} / J) ~ m i c e ~ w e r e ~ p u r c h a s e d ~ f r o m ~}$ the Jackson Laboratory and NF-kB-RE-luc [BALB/c-Tg(Rela-luc)31Xen] transgenic mice were purchased from Taconic. Traf1 ${ }^{-1-}$ mice were a gift from Tania Watts (University of Toronto, Toronto, Canada) and were

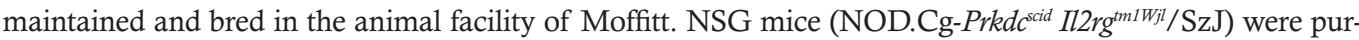
chased from Jackson Laboratory and bred in the animal facility of Moffitt. Female and/or male mice at 8-12 weeks of age were used for the study. For survival studies, mice were injected i.v. with E $\mu$-ALL $\left(1 \times 10^{6}\right.$ cells $/$ mouse, day 0), followed by i.p. cyclophosphamide (250-300 mg/kg, days 6-7) and mCD19-targeted CAR T cells $\left(0.15 \times 10^{6}\right.$ to $5 \times 10^{6} \mathrm{CAR} \mathrm{T}$ cells/mouse, days 7-10). Mice were monitored for illness and sacrificed when there was evidence of leukemia progression, such as decreased activity, hunched posture, and ruffled

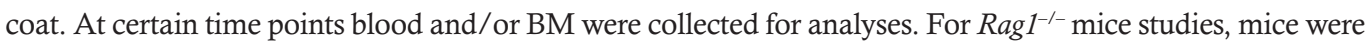
i.v. injected with $1 \times 10^{6} \mathrm{mCD} 19$-targeted CAR T cells. Blood and BM were collected for flow cytometry.

Cells. The E $\mu$-ALL cell line has been described previously (26). The cells were cultured with irradiated (30 Gy) NIH/3T3 fibroblasts as feeders. The culture medium consists of an equal volume of (a) IMDM supplemented with $2 \mathrm{mM} \mathrm{L}$-glutamine, $55 \mu \mathrm{M} \beta$-mercaptoethanol, $100 \mathrm{U} / \mathrm{ml}$ penicillin, $100 \mu \mathrm{g} / \mathrm{ml}$ streptomycin, and 
10\% FBS; and (b) DMEM supplemented with $2 \mathrm{mM}$ L-glutamine, $100 \mathrm{U} / \mathrm{ml}$ penicillin, $100 \mu \mathrm{g} / \mathrm{ml}$ streptomycin, and 10\% calf serum. EL4-mCD19 cells were used as target cells and have been described previously (26). 3T3-mCD19 and 3T3-hCD19 cells are NIH/3T3 cells retrovirally transduced with mouse or human CD19 and were used as target cells. $\mathrm{CHO}-\mathrm{hCD} 33$ cells are Chinese hamster ovary $(\mathrm{CHO})$ cells retrovirally transduced with human CD33 and were used as target cells for human CD33-targeted CAR T cells. NIH/3T3 and CHO cells were purchased from ATCC. Mouse T cell complete medium consists of RPMI1640 medium, 10\% FBS, $1 \mathrm{mM}$ sodium pyruvate, $1 \times$ NEAA (non-essential amino acids), $10 \mathrm{mM}$ HEPES, $55 \mu \mathrm{M} \beta$-mercaptoethanol, $2 \mathrm{mM}$ L-glutamine, $100 \mathrm{U} / \mathrm{ml}$ penicillin, and $100 \mu \mathrm{g} / \mathrm{ml}$ streptomycin. Human peripheral blood mononuclear cells from healthy donors were purchased from ReachBio. Human T cell complete medium consists of RPMI1640 medium, 10\% FBS, 2 mM L-glutamine, $100 \mathrm{U} / \mathrm{ml}$ penicillin, and $100 \mu \mathrm{g} / \mathrm{ml}$ streptomycin. All media and supplements were from ThermoFisher Scientific. NF-кB/293/GFP-Luc Transcriptional Reporter Cells were purchased from System Biosciences, maintained and used according to the manufacturer's instructions. FFLuc-GFP NALM6 (NALM6-GL) cells have been described previously (53).

Genetic constructs and CAR T cell production. The SFG retroviral construct was used for all constructs and $\mathrm{m} 19 \Delta \mathrm{z}, \mathrm{m} 19 \mathrm{z}, \mathrm{m} 1928 \mathrm{z}$, and $\mathrm{m} 19-\mathrm{musBBz}$ CAR have been described previously $(26,54)$. We modified these constructs to replace the mouse 4-1BB endodomain with human 4-1BB endodomain or mutated mouse 4-1BB domain (Figure 2A and Supplemental Figure 2A). An hCD19-targeted CAR was synthesized by GENEWIZ to include the FMC63 scFv combined with human counterparts to the mouse 4-1BB endodomain listed in Supplemental Figure 2A. TRAF and TRAF DN constructs include the coding sequences, glycine serine linker, cerulean, and stop codon, which were synthesized and subcloned into the SFG retroviral vector. TRAF DN coding sequences have been described previously (55). TRAF1 DN (aa 184-417) consists only of the TRAF domain, TRAF2 DN (aa 87-501) lacks the ring finger domain, and TRAF3 DN (aa 382-568) also lacks the ring finger domain.

All SFG constructs were calcium phosphate transfected into H29 cells. Retroviral supernatants of transfected H29 cells were harvested and used to transduce Phoenix E cells for mouse T cell transduction or RD114 cells for human T cell transduction. Retroviral supernatant of Phoenix E or RD114 producer cells were harvested, $0.45-\mu \mathrm{m}$ filtered, and used to transduce mouse or human $\mathrm{T}$ cells as described previously $(26,56)$. For TRAF-overexpressing CAR T cells, T cells were cotransduced with retrovirus containing CAR or TRAF at day 1 and day 2 . At day 3 or day 4 CAR T cells were collected, beads removed, and subjected to counting and viability evaluation before downstream experimental use. Viability was measured by staining cells with trypan blue and enumerated on an automated cell counter (Bio-Rad). Transduction efficiency was estimated as percentage of $\mathrm{GFP}^{+}$or Cherry ${ }^{+}$live cells as detected by flow cytometry. In some experiments, CAR expression was evaluated by staining $\mathrm{T}$ cells with $1 \mu \mathrm{g}$ Biotin-Protein L (GenScript) followed by fluorochrome-conjugated streptavidin (eBioscience) and flow cytometry as described previously (57). For downstream experiments, CAR T cell doses were normalized based on CAR gene transfer but not sorted to exclude CAR-negative T cells, so the total T cell dose varied. For the irradiation study, CAR T cells were irradiated at $10 \mathrm{~Gy}$. Development of the CD33-targeted CARs are described in supplemental methods.

Flow cytometry. The following anti-mouse or anti-human antibodies with clones listed were obtained from eBioscience: anti-mCD16/CD32 (clone 93), anti-mB220 (RA3-6B2), anti-mCD19 (eBio1D3), antimCD3 (145-2C11), anti-mCD4 (GK1.5), anti-mCD8 (53-6.7), anti-mThy1.1 (HIS51), anti-mCD44 (1M7), anti-mCD62L (MEL-14), anti-mTER119 (TER-119), anti-mCD11b (M1/70), anti-mGr1 (RB6-8C5), antimNK1.1 (PK136), anti-mIFN- $\gamma$ (XMG1.2), anti-mTNF- $\alpha$ (MP6-XT22), and anti-mBCL2 (10C4). The following antibodies were from Biolegend: anti-mCD3 (17A2), anti-mCD4 (RM4-5), anti-mCD8 (53-6.7). The following antibodies were from BD Bioscience: anti-hCD3 (UCHT1), anti-hCD4 (SK3), and antihCD8 (RAP-T8). Anti-BCL-XL (54H6) was from Cell Signaling Technology.

Cells were first washed twice with PBS and stained with fixable viability dye (eBioscience). Surface staining was performed at $4^{\circ} \mathrm{C}$ with $\mathrm{Fc}$ block (eBioscience) and antibody mix in MACS buffer with $0.5 \%$ BSA (Miltenyi Biotec). For intracellular staining, $1 \times 10^{6}$ CAR T cells were cocultured with $1 \times 10^{5}$ irradiated 3T3-mCD19 for 4 hours in the presence of protein transport inhibitor (eBioscience). T cells were harvested, fixed, and permeabilized with Intracellular Fixation and Permeabilization Buffer Set (eBioscience) followed by antibody staining. The manufacturer's instructions were followed. Peripheral blood samples were stained with antibodies and lysed afterwards using BD FACS lysing solution (26). For some experiments, Countbright beads (ThermoFisher Scientific) were used for cell quantification. 
All samples were analyzed with a 5-laser BD LSRII (BD Biosciences) and data were analyzed using FlowJo software (Tree Star).

Cytokine immunoassay. One million mouse CAR T cells were cocultured with $1 \times 10^{5}$ 3T3-mCD19 cells for 24 hours. Supernatants were harvested and analyzed using a Mouse Luminex Assay kit (R\&D Systems). Data were collected on a Luminex 100 system (Luminex). The manufacturer's instructions were followed. For human CAR T cell study, CAR T cells were cocultured with 3T3-hCD19 cells at 10:1 for 24 hours. Supernatants were harvested and analyzed using a Simple Plex Assay Kit (R\&D Systems) on an Ella machine (ProteinSimple). Manufacturers' instructions were followed.

Cytotoxicity assay. A 4-hour chromium-release assay was performed with EL4-mCD19 as target cells and mCD19-targeted CAR T cells as effectors. Our methods have been described previously (26). Cytotoxicity assays were also run on an xCELLigence RTCA (real-time cell analysis) instrument (ACEA Biosciences) according to the manufacturer's instructions. Briefly, 3T3-mCD19 or 3T3-hCD19 cells were seeded at 10,000 cells per well in an E-Plate 96. On the next day, mouse or human CAR T cells were resuspended in fresh complete medium without IL-2 and added onto target cells at different E/T ratios and cell growth was monitored.

Western blot and immunoprecipitation. CAR T cells were stimulated with 3T3-mCD19 at a 10:1 ratio for 4 hours. Cell lysates were prepared using $240 \mu 1$ of cell lysis buffer (Cell Signaling Technology) for $6 \times$ $10^{6} \mathrm{CAR} \mathrm{T}$ cells. Thirty microliters of reduced and denatured cell lysates were electrophoresed through a 10\% Mini-PROTEAN TGX Precast gel (Bio-Rad), transferred to nitrocellulose blot membranes, blocked, and the membranes were cut based on molecular weight to probe different proteins. The membranes were incubated with primary antibody at 1:1,000 overnight at $4^{\circ} \mathrm{C}$. Blots were washed and incubated with HRPlinked anti-rabbit IgG (Cell Signaling Technology) at 1:10,000 for 1 hour at room temperature. Blots were washed again and incubated with SuperSignal West Femto Maximum Sensitivity Substrate (ThermoFisher Scientific). Images were acquired on an Odyssey Fc imaging system (LI-COR Biotechnology). Protein semiquantification was achieved by using ImageJ software (NIH). Anti-BCL-XL (54H6), anti-BCL2 (D17C4), and anti- $\beta$-actin rabbit mAb (13E5) were from Cell Signaling Technology.

For immunoprecipitation experiments, $30 \times 10^{6}$ CAR-expressing NF- $\mathrm{BB} / 293 /$ GFP-Luc reporter cells were lysed using RIPA buffer (Cell Biolabs, Inc) supplemented with cOmplete Protease Inhibitor Cocktail (Sigma-Aldrich), following the manufacturer's recommendations. Protein extracts were incubated with Pierce Protein-L magnetic beads (ThermoFisher Scientific) overnight at $4^{\circ} \mathrm{C}$. Immune complexes were recovered using a DynaMag-2 magnet (Life Technologies), and prepared for SDS-PAGE.

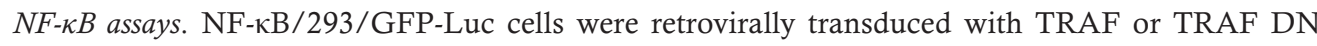
constructs and CD19-targeted CARs. NF- $\mathrm{BB}$ signaling was evaluated by measuring GFP expression with flow cytometry. CAR T cells were generated from NF- $\mathrm{kB}-\mathrm{RE}-\mathrm{Luc}$-transgenic splenocytes. CAR T cells were cocultured with irradiated (30 Gy) 3T3-mCD19 cells in 6-well plates for 4 hours. For each group, T cells, normalized to $3 \times 10^{6} \mathrm{mCD} 19$-targeted CAR T cells per well, were incubated with $3 \times 10^{5}$ 3T3-mCD19 cells per well. After stimulation, cell lysates were prepared using Cell Culture Lysis Reagent (Promega). Luciferase assay was performed using a luciferase assay kit (Promega) according to the manufacturer's instructions. Cell lysates were added at $20 \mu 1$ per well in a 96-well white plate (Corning), followed by $100 \mu \mathrm{l}$ of Luciferase Assay Reagent per well, and bioluminescence was immediately measured on a SpectraMax L microplate luminometer (Molecular Devices). Each sample was assayed in triplicate.

hCD19-targeted CAR T cell in vitro proliferation. Normalized numbers $\left(1\right.$ or $\left.2 \times 10^{6}\right)$ of human CAR T cells were cocultured with $2 \times 10^{5} 3 \mathrm{~T} 3$-hCD19 AAPCs per well in non-tissue-culture-treated 6-well plates in triplicate. Cells were grown in human T cell complete medium supplemented with $60 \mathrm{IU} / \mathrm{ml} \mathrm{IL}-2$ and split every 2 to 3 days or whenever the medium turned yellow. Cell viability and total cell numbers in each well were measured daily or every 2 to 4 days ( $\mathrm{T}$ isolation as day 0 ) on a cell counter (Bio-Rad) with trypan blue staining. For flow cytometric analysis of in vitro proliferation, CAR T cells were stained with eFluor670 proliferation dye (eBioscience) and then cocultured with target cells at a 5:1 ratio for 4 days.

Gene expression analysis. Complete descriptions of the microarray, RNA-Seq, and differential gene expression analyses used in this study are provided in the supplemental methods. Microarray and RNA-Seq data were deposited in the NCBI's Gene Expression Omnibus database (GEO GSE112567).

Statistics. Means were compared using a 2-sided unpaired parametric $t$ test. Cytotoxicity curves were compared using a Kolmogorov-Smirnov test. Human CAR T cell in vitro proliferation was compared using 2-way ANOVA. Survival was compared using a log-rank test. Statistical analyses were conducted using GraphPad Prism software 7 and the R software package (https://www.r-project.org). $P<0.05$ was considered significant. 
Study approval. Animal studies were performed under protocols approved by the Institutional Animal Care and Use Committee of Vanderbilt University and the University of South Florida.

\section{Author contributions}

GL and MLD designed the experiments and wrote the manuscript. GL, JCB, HK, KP, YZ, BS, LG, NB, $\mathrm{XW}$, and DAD conducted experiments, acquired data, analyzed data, and assisted with the manuscript.

\section{Acknowledgments}

This work was supported with funds from the H. Lee Moffitt Cancer Center and Research Institute (to MLD), as well as a Damon Runyon Clinical Investigator award (to MLD), and ASH Amos Medical Faculty Development award (to MLD). We are grateful for the assistance of the Flow Cytometry Shared Resource and Vanderbilt Technologies for Advanced Genomics (VANTAGE) at Vanderbilt University Medical Center. Our study received assistance from the Flow Cytometry Core Facility and the Molecular Genomics and Cancer Informatics Core Facilities at the H. Lee Moffitt Cancer Center \& Research Institute, an NCI-designated Comprehensive Cancer Center, supported under NIH grant P30-CA76292. The authors would like to acknowledge the animal care staff in the Department of Comparative Medicine at the University of South Florida for providing technical assistance. We are also thankful to Jose ConejoGarcia and Claudio Anasetti for assistance with manuscript preparation and Scott Antonia, James Mule, and John Cleveland for data discussion.

Address correspondence to: Marco Luis Davila, 12902 Magnolia Drive, Tampa, Florida, 33612, USA. Phone: 813.745.7202; Email: marco.davila@moffitt.org.

KP's present address is: Department of Cell and Developmental Biology, School of Medicine, Vanderbilt University, Nashville, Tennessee, USA.

1. Rosenberg SA, et al. Use of tumor-infiltrating lymphocytes and interleukin-2 in the immunotherapy of patients with metastatic melanoma. A preliminary report. NEngl J Med. 1988;319(25):1676-1680.

2. Tran E, et al. Cancer immunotherapy based on mutation-specific $\mathrm{CD} 4^{+} \mathrm{T}$ cells in a patient with epithelial cancer. Science. 2014;344(6184):641-645.

3. Morgan RA, et al. Cancer regression in patients after transfer of genetically engineered lymphocytes. Science. 2006;314(5796):126-129.

4. Porter DL, Levine BL, Kalos M, Bagg A, June CH. Chimeric antigen receptor-modified T cells in chronic lymphoid leukemia. N Engl J Med. 2011;365(8):725-733.

5. Eshhar Z, Waks T, Gross G, Schindler DG. Specific activation and targeting of cytotoxic lymphocytes through chimeric single chains consisting of antibody-binding domains and the gamma or zeta subunits of the immunoglobulin and T-cell receptors. Proc Natl Acad Sci USA. 1993;90(2):720-724.

6. Brocker T. Chimeric Fv-zeta or Fv-epsilon receptors are not sufficient to induce activation or cytokine production in peripheral T cells. Blood. 2000;96(5):1999-2001.

7. Brentjens RJ, et al. Eradication of systemic B-cell tumors by genetically targeted human T lymphocytes co-stimulated by CD 80 and interleukin-15. Nat Med. 2003;9(3):279-286.

8. Gong MC, Latouche JB, Krause A, Heston WD, Bander NH, Sadelain M. Cancer patient T cells genetically targeted to prostate-specific membrane antigen specifically lyse prostate cancer cells and release cytokines in response to prostate-specific membrane antigen. Neoplasia. 1999;1(2):123-127.

9. Finney HM, Lawson AD, Bebbington CR, Weir AN. Chimeric receptors providing both primary and costimulatory signaling in T cells from a single gene product. J Immunol. 1998;161(6):2791-2797.

10. Maher J, Brentjens RJ, Gunset G, Rivière I, Sadelain M. Human T-lymphocyte cytotoxicity and proliferation directed by a single chimeric TCRzeta /CD28 receptor. Nat Biotechnol. 2002;20(1):70-75.

11. Imai $C$, et al. Chimeric receptors with $4-1 \mathrm{BB}$ signaling capacity provoke potent cytotoxicity against acute lymphoblastic leukemia. Leukemia. 2004;18(4):676-684.

12. Finney HM, Akbar AN, Lawson AD. Activation of resting human primary T cells with chimeric receptors: costimulation from CD28, inducible costimulator, CD134, and CD137 in series with signals from the TCR zeta chain. J Immunol. 2004;172(1):104-113.

13. Milone MC, et al. Chimeric receptors containing CD137 signal transduction domains mediate enhanced survival of T cells and increased antileukemic efficacy in vivo. Mol Ther. 2009;17(8):1453-1464.

14. Davila ML, et al. Efficacy and toxicity management of 19-28z CAR T cell therapy in B cell acute lymphoblastic leukemia. Sci Transl Med. 2014;6(224):224ra25.

15. Maude SL, et al. Chimeric antigen receptor T cells for sustained remissions in leukemia. N Engl J Med. 2014;371(16):1507-1517.

16. Lee DW, et al. T cells expressing CD19 chimeric antigen receptors for acute lymphoblastic leukaemia in children and young adults: a phase 1 dose-escalation trial. Lancet. 2015;385(9967):517-528.

17. Turtle CJ, et al. CD19 CAR-T cells of defined $\mathrm{CD} 4^{+}: \mathrm{CD} 8^{+}$composition in adult B cell ALL patients. J Clin Invest. 
2016;126(6):2123-2138.

18. Turtle CJ, et al. Immunotherapy of non-Hodgkin's lymphoma with a defined ratio of CD8 ${ }^{+}$and CD4 ${ }^{+} \mathrm{CD} 19$-specific chimeric antigen receptor-modified T cells. Sci Transl Med. 2016;8(355):355ra116.

19. Kochenderfer JN, et al. Chemotherapy-refractory diffuse large B-cell lymphoma and indolent B-cell malignancies can be effectively treated with autologous T cells expressing an anti-CD19 chimeric antigen receptor. J Clin Oncol. 2015;33(6):540-549.

20. Park JH, et al. Long-term follow-up of CD19 CAR therapy in acute lymphoblastic leukemia. N Engl J Med. 2018;378(5):449-459.

21. Neelapu SS, et al. Axicabtagene ciloleucel CAR T-cell therapy in refractory large B-cell lymphoma. $N$ Engl J Med. 2017;377(26):2531-2544.

22. Maude SL, et al. Tisagenlecleucel in children and young adults with B-cell lymphoblastic leukemia. N Engl J Med. 2018;378(5):439-448.

23. Ramos CA, et al. Clinical responses with $\mathrm{T}$ lymphocytes targeting malignancy-associated $\kappa$ light chains. J Clin Invest. 2016;126(7):2588-2596.

24. Sabbagh L, Pulle G, Liu Y, Tsitsikov EN, Watts TH. ERK-dependent Bim modulation downstream of the 4-1BB-TRAF1 signaling axis is a critical mediator of CD8 T cell survival in vivo. J Immunol. 2008;180(12):8093-8101.

25. Zarnegar BJ, et al. Noncanonical NF-kappaB activation requires coordinated assembly of a regulatory complex of the adaptors cIAP1, cIAP2, TRAF2 and TRAF3 and the kinase NIK. Nat Immunol. 2008;9(12):1371-1378.

26. Davila ML, Kloss CC, Gunset G, Sadelain M. CD19 CAR-targeted T cells induce long-term remission and B Cell Aplasia in an immunocompetent mouse model of B cell acute lymphoblastic leukemia. PLoS ONE. 2013;8(4):e61338.

27. Zhao Z, et al. Structural design of engineered costimulation determines tumor rejection kinetics and persistence of CAR T cells Cancer Cell. 2015;28(4):415-428.

28. Walker AJ, et al. Tumor antigen and receptor densities regulate efficacy of a chimeric antigen receptor targeting anaplastic lymphoma kinase. Mol Ther. 2017;25(9):2189-2201.

29. Jang IK, Lee ZH, Kim YJ, Kim SH, Kwon BS. Human 4-1BB (CD137) signals are mediated by TRAF2 and activate nuclear factor-kappa B. Biochem Biophys Res Commun. 1998;242(3):613-620.

30. Arch RH, Thompson CB. 4-1BB and Ox40 are members of a tumor necrosis factor (TNF)-nerve growth factor receptor subfamily that bind TNF receptor-associated factors and activate nuclear factor kappaB. Mol Cell Biol. 1998;18(1):558-565.

31. McPherson AJ, Snell LM, Mak TW, Watts TH. Opposing roles for TRAF1 in the alternative versus classical NF-kB pathway in T cells. J Biol Chem. 2012;287(27):23010-23019.

32. Saoulli K, et al. CD28-independent, TRAF2-dependent costimulation of resting T cells by 4-1BB ligand. J Exp Med. 1998;187(11):1849-1862.

33. Ye H, Park YC, Kreishman M, Kieff E, Wu H. The structural basis for the recognition of diverse receptor sequences by TRAF2 Mol Cell. 1999;4(3):321-330.

34. Vallabhapurapu S, et al. Nonredundant and complementary functions of TRAF2 and TRAF3 in a ubiquitination cascade that activates NIK-dependent alternative NF-kappaB signaling. Nat Immunol. 2008;9(12):1364-1370.

35. Lee HW, Park SJ, Choi BK, Kim HH, Nam KO, Kwon BS. 4-1BB promotes the survival of CD8 ${ }^{+} \mathrm{T}$ lymphocytes by increasing expression of Bcl-xL and Bfl-1. J Immunol. 2002;169(9):4882-4888.

36. Watts TH. TNF/TNFR family members in costimulation of T cell responses. Annu Rev Immunol. 2005;23:23-68.

37. Barnes SE, et al. T cell-NF-кB activation is required for tumor control in vivo. J Immunother Cancer. 2015;3(1):1.

38. Carlsen H, Moskaug JØ, Fromm SH, Blomhoff R. In vivo imaging of NF-kappa B activity. J Immunol. 2002;168(3):1441-1446.

39. Gomes-Silva D, et al. Tonic 4-1BB costimulation in chimeric antigen receptors impedes $\mathrm{T}$ cell survival and is vector-dependent. Cell Rep. 2017;21(1):17-26.

40. Tsitsikov EN, et al. TRAF1 is a negative regulator of TNF signaling. enhanced TNF signaling in TRAF1-deficient mice. Immunity. 2001;15(4):647-657.

41. [No authors listed]Value in using CAR T cells for DLBCL. Cancer Discov. 2018;8(2):131-132.

42. Brentjens RJ, et al. Genetically targeted T cells eradicate systemic acute lymphoblastic leukemia xenografts. Clin Cancer Res. 2007;13(18 Pt 1):5426-5435

43. Zhong XS, Matsushita M, Plotkin J, Riviere I, Sadelain M. Chimeric antigen receptors combining 4-1BB and CD28 signaling domains augment PI3kinase/AKT/Bcl-XL activation and CD8 ${ }^{+}$T cell-mediated tumor eradication. Mol Ther. 2010;18(2):413-420.

44. Yang Y, et al. TCR engagement negatively affects CD8 but not CD4 CAR T cell expansion and leukemic clearance. Sci Transl Med. 2017;9(417):eaag1209.

45. Kawalekar OU, et al. Distinct signaling of coreceptors regulates specific metabolism pathways and impacts memory development in CAR T cells. Immunity. 2016;44(2):380-390.

46. Long AH, et al. 4-1BB costimulation ameliorates $\mathrm{T}$ cell exhaustion induced by tonic signaling of chimeric antigen receptors. Nat Med. 2015;21(6):581-590.

47. Knudson KM, Pritzl CJ, Saxena V, Altman A, Daniels MA, Teixeiro E. NFкB-Pim-1-Eomesodermin axis is critical for maintaining CD8 T-cell memory quality. Proc Natl Acad Sci USA. 2017;114(9):E1659-E1667.

48. Mauro $\mathrm{C}$, et al. NF- $\mathrm{KB}$ controls energy homeostasis and metabolic adaptation by upregulating mitochondrial respiration. Nat Cell Biol. 2011;13(10):1272-1279.

49. So T, Nagashima H, Ishii N. TNF receptor-associated factor (TRAF) signaling network in CD4(+) T-lymphocytes. Tohoku J Exp Med. 2015;236(2):139-154.

50. Xie P, Kraus ZJ, Stunz LL, Liu Y, Bishop GA. TNF receptor-associated factor 3 is required for T cell-mediated immunity and TCR/CD28 signaling. J Immunol. 2011;186(1):143-155.

51. Till BG, et al. CD20-specific adoptive immunotherapy for lymphoma using a chimeric antigen receptor with both CD28 and 4-1BB domains: pilot clinical trial results. Blood. 2012;119(17):3940-3950.

52. Park MH, Hong JT. Roles of NF- $\mathrm{kB}$ in cancer and inflammatory diseases and their therapeutic approaches. Cells. 2016;5(2).

53. Zhao Z, et al. Structural design of engineered costimulation determines tumor rejection kinetics and persistence of CAR $\mathrm{T}$ cells. Cancer Cell. 2015;28(4):415-428.

54. Ghosh A, et al. Donor CD19 CAR T cells exert potent graft-versus-lymphoma activity with diminished graft-versus-host activity. 
Nat Med. 2017;23(2):242-249.

55. Duckett CS, Gedrich RW, Gilfillan MC, Thompson CB. Induction of nuclear factor kappaB by the CD30 receptor is mediated by TRAF1 and TRAF2. Mol Cell Biol. 1997;17(3):1535-1542.

56. Li G, Park K, Davila ML. Gammaretroviral production and $\mathrm{T}$ cell transduction to genetically retarget primary $\mathrm{T}$ cells against cancer. Methods Mol Biol. 2017;1514:111-118.

57. Zheng Z, Chinnasamy N, Morgan RA. Protein L: a novel reagent for the detection of chimeric antigen receptor (CAR) expression by flow cytometry. J Transl Med. 2012;10:29. 